\title{
The inner solar system cratering record and the evolution of impactor populations
}

\author{
Robert G. Strom ${ }^{1}$, Renu Malhotra ${ }^{1}$, Zhiyong Xiao ${ }^{2}$, Takashi Ito ${ }^{3}$, Fumi Yoshida ${ }^{3}$, Lillian R. \\ Ostrach $^{4}$ \\ ${ }^{1}$ Lunar and Planetary Laboratory, The University of Arizona, Tucson, Arizona 85721, USA; \\ rstrom@lpl.arizona.edu \\ ${ }^{2}$ Faculty of Earth Sciences, China University of Geosciences (Wuhan), Wuhan, Hubei, China \\ 3 National Astronomical Observatory, Osawa 2-21-1, Mitaka, Tokyo 181-8588, Japan \\ ${ }^{4}$ School of Earth and Space Exploration, Arizona State University, Tempe, Arizona, USA \\ Received 20XX XXXX XX; accepted 20XX XXXX XX
}

\begin{abstract}
We review previously published and newly obtained crater size-frequency distributions in the inner solar system. These data indicate that the Moon and the terrestrial planets have been bombarded by two populations of objects. Population 1, dominating at early times, had nearly the same size distribution as the present-day asteroid belt, and produced the heavily cratered surfaces with a complex, multi-sloped crater size-frequency distribution. Population 2, dominating since about 3.8-3.7 Ga, has the same size distribution as near-Earth objects (NEOs), had a much lower impact flux, and produced a crater size distribution characterized by a differential -3 single-slope power law in the crater diameter range $0.02 \mathrm{~km}$ to $100 \mathrm{~km}$. Taken together with the results from a large body of work on age-dating of lunar and meteorite samples and theoretical work in solar system dynamics, a plausible interpretation of these data is as follows. The NEO population is the source of Population 2 and it has been in near-steady state over the past $\sim 3.7-3.8$ gigayears; these objects are derived from the main asteroid belt by size-dependent non-gravitational effects that favor the ejection of smaller asteroids. However, Population 1 were main belt asteroids ejected from their source region in a size-independent manner, possibly by means of gravitational resonance sweeping during giant planet orbit migration; this caused the so-called Late Heavy Bombardment (LHB). The LHB began some time before $\sim 3.9 \mathrm{Ga}$, peaked and declined rapidly over the next $\sim 100$ to 300 megayears, and possibly more slowly from about $3.8-3.7 \mathrm{Ga}$ to $\sim 2 \mathrm{Ga}$. A
\end{abstract}


third crater population (Population S) consists of secondary impact craters that can dominate the cratering record at small diameters.

Key words: solar system: formation - minor planets, asteroids — Earth - Moon

\section{INTRODUCTION}

It is often implied that there is only one crater population in the Solar system caused by one population of impacting objects. Some authors (e.g. Hartmann, 1995, Hartmann \& Neukum, 2001, Ivanov et al., 2002) maintain that the crater size-frequency distribution (SFD) characteristic of the ancient period of heavy bombardment has persisted throughout Solar system history. The commonly used scheme for determining absolute model ages derived from the impact cratering record is based on this assumption (e.g. Neukum et al., 2001; Hartmann, 2005; Michael, 2013).

Based on crater SFDs, however, Strom et al. (2005) suggested that the inner Solar system planets and the Moon have been bombarded by two different populations of objects, distinguishable by their sizefrequency distributions. Following Strom et al. (2005), we refer to these as Population 1 and Population 2. Population 1 is associated with the heavily cratered surfaces on Mercury, the Moon, and Mars; it has a complex size distribution of craters, characterized by a differential -2.2 slope at diameters less than about $50 \mathrm{~km}$, a nearly flat part (differential slope -3 ) between 50 and $100 \mathrm{~km}$, and sloping downward to the right (differential slope -4 ) at diameters between about $100 \mathrm{~km}$ to $300 \mathrm{~km}$. It also appears to slope upward at diameters between about 400 and $1000 \mathrm{~km}$. Population 2 is associated primarily with lightly cratered, younger plains units on the Moon, Venus, Mars, and Mercury; these craters are characterized by a singleslope differential -3 power-law size distribution which plots as a nearly straight horizontal line on an $R$ plot. The crater density of Population 1 exceeds that of Population 2 by more than an order of magnitude for crater diameters greater than $10 \mathrm{~km}$. Figure 1 shows the Relative plots (“ $R$ plots", see section 2] of examples of these two crater populations.

The much higher density of Population 1 indicates that it is a much older impact record than Population 2. The differing shapes of the two populations suggest the possibility of different dynamical origins of the Population 1 (old) and Population 2 (younger) impactor populations. The absence of Population 1 on younger terrains implies that the SFD of inner solar system impactors changed from Population 1 to Population 2 at some ancient time.

Distinguishing between the two crater populations and understanding the time evolution of the impactor populations is important to understanding the dynamical history of the Solar system. It is also directly related to the reliability of the prevalent age dating technique using crater counts (Michael \& Neukum, 2010). Many of the crater SFDs published in various journals and book chapters over the past $\sim 40$ years were presented before it was realized that two crater populations were present on the Moon and the terrestrial planets, and that they differed significantly from crater populations on the outer planet satellites. During 


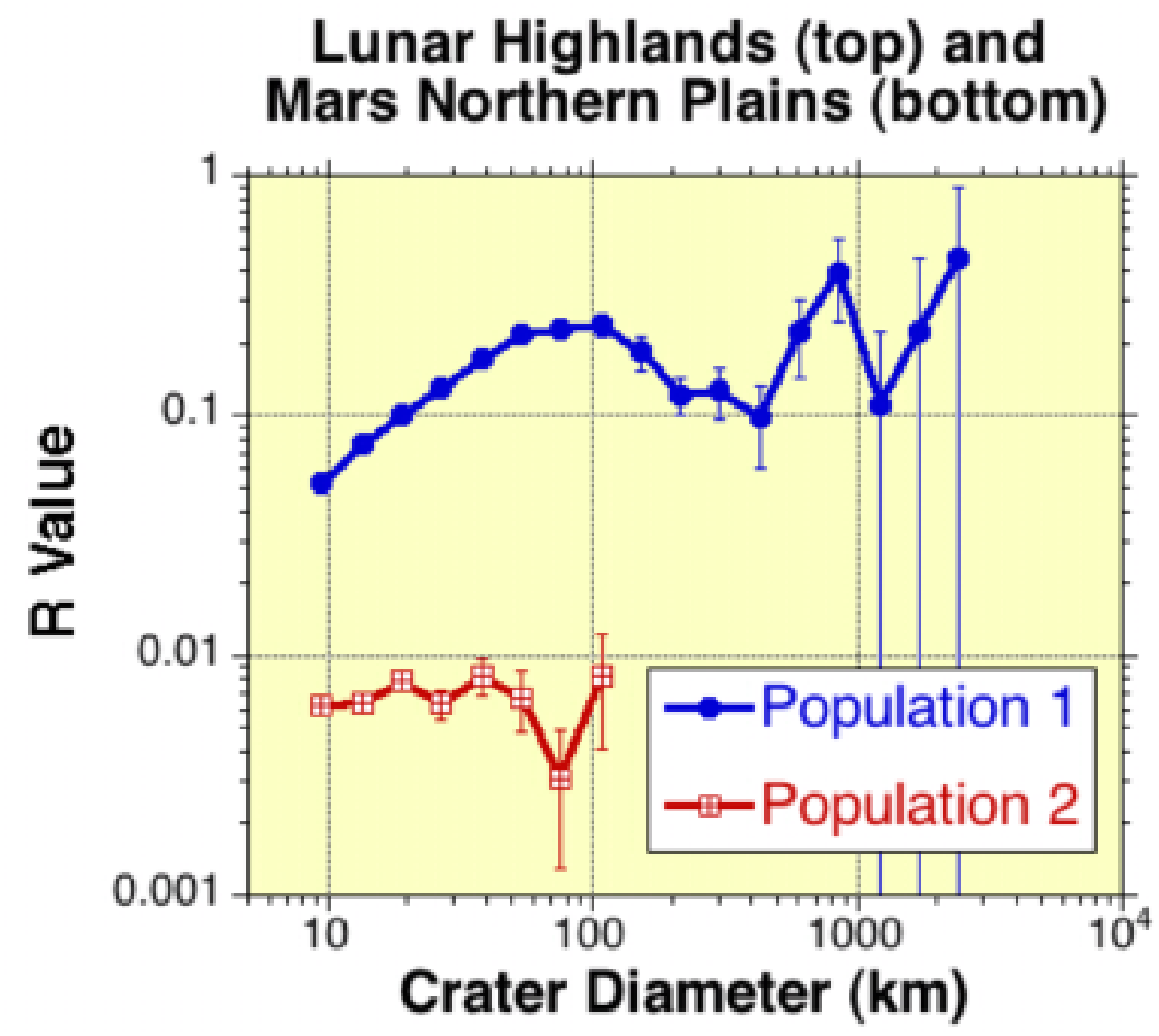

Fig. 1 This $R$ plot shows the complex SFD characteristic of Population 1 craters, represented here by the lunar highlands craters, and the nearly horizontal curve (equivalently, a simple -3 power law of the SFD) of Population 2 craters represented here by the craters on the Northern Plains of Mars.

most of this time the origin of the objects responsible for the cratering record was not well understood. Also, it was not clearly understood how widespread and important were secondary impact craters to the small crater population, at diameters $D \lesssim 1 \mathrm{~km}$ on the Moon and Mars and $\lesssim 10 \mathrm{~km}$ on Mercury.

In the present paper we review and provide an updated and detailed record of the data on the two crater populations and an updated discussion of their implications for the time evolution of impactor populations in the inner solar system; these were presented only in abbreviated summary form in Strom et al. (2005). We also present newly obtained MESSENGER data for craters on Mercury, new crater counts on the Orientale basin on the Moon and new counts of small rayed craters on Mars. These new data and an analysis of secondary impact craters augment and support the previously published crater SFDs found on the inner solar system planets.

This paper is organized as follows. In Section 2 we describe in some detail the definition and usefulness of the $R$ plot. In Section 3, we give a comprehensive summary of the crater SFDs found on each of the 
terrestrial planets and the Moon, as well a discussion of the secondary crater data; as a foil to the inner solar system crater record, we also provide (in section 3.4) a summary of the crater SFDs found on outer planet satellites. In Section 4, we discuss the implications of the crater record and its interpretation in theoretical models of the dynamical history of the solar system, including the putative spike in the impact flux known as the 'Late Heavy Bombardment' (LHB) that is thought to have occurred at about $\sim 3.9$ Ga (Turner et al. 1973: Tera et al., 1974). A list of references and notes for our sources of data as well as two supplementary figures are provided in the Appendix.

\section{THE RELATIVE SIZE-FREQUENCY DISTRIBUTION PLOT}

The "Relative" plot (or $R$ plot) method of displaying the crater and projectile SFDs is used throughout this paper. The $R$ plot was devised by the Crater Analysis Techniques Working Group (Crater analysis techniques working group, 1979) to better show the size distribution of craters and crater number densities for determining relative ages. When sufficiently large and accurate data sets are available, the $R$ plot provides a more critical and sensitive comparison between SFDs than cumulative plots. The latter tend to smear out important details of the crater SFD curves and can lead to erroneous interpretations. This happened frequently in the 1960s and 1970s, which led to the formation of a NASA Working Group to remedy the problem. Figure 2 is a diagrammatic representation of a differential -2 and -3 size distribution for diameters between 11 and $64 \mathrm{~km}$ diameter. This figure illustrates visually the large difference between differential -2 and -3 power law distributions. The significant differences between these distributions were sometimes overlooked owing to the use of cumulative plots (e.g., Hartmann (1966); Wilhelms et al. (1978); Michael (2013)). The $R$ plot was strongly recommended by the Crater Analysis Techniques Working Group (Crater analysis techniques working group, 1979), in addition to any other plots authors chose to use.

On an $R$ plot, the SFD is normalized to a power law differential size distribution function, $d N(D) \sim$ $D^{p} d D$, where $D$ is diameter. The index $p=-3$ is recommended because most crater SFDs are observed to be piecewise within \pm 1 of a $p=-3$ power law distribution. The discretized equation for the $R$ value is: $R=D^{3} N / A\left(b_{2}-b_{1}\right)$; where $D$ is the geometric mean diameter of the size bin $\left.\sqrt{b_{1} b_{2}}\right), N$ is the number of craters in the size bin, $A$ is the area over which the counts were made, $b_{1}$ is the lower limit of the size bin, and $b_{2}$ is the upper limit of the size bin. Usually, although not required, the size bins are in $\sqrt{2}$ increments because there are many more small craters than large craters. In an $R$ plot, $\log _{10} R$ is plotted on the $y$-axis and $\log _{10} D$ is plotted on the $x$-axis. Thus, a $p=-3$ SFD plots as a horizontal straight line; a $p=-2$ SFD slopes down to the left at an angle of $45^{\circ}$, and a $p=-4$ SFD slopes down to the right at $45^{\circ}$. Differences in the shapes of the curves can be either due to differences in the properties of the impactor populations or to differences in target properties. The vertical position of the curve is a measure of crater density, hence relative age, on the same planet: the higher the vertical position, the higher the crater density and the older the surface. For comparisons amongst different planets, differences in impact fluxes, impact velocities and target properties must be taken into account when using crater densities to infer relative ages. 


\section{Mercury, Moon, and Mars Highlands (Dia. $<50 \mathrm{~km}$ )}
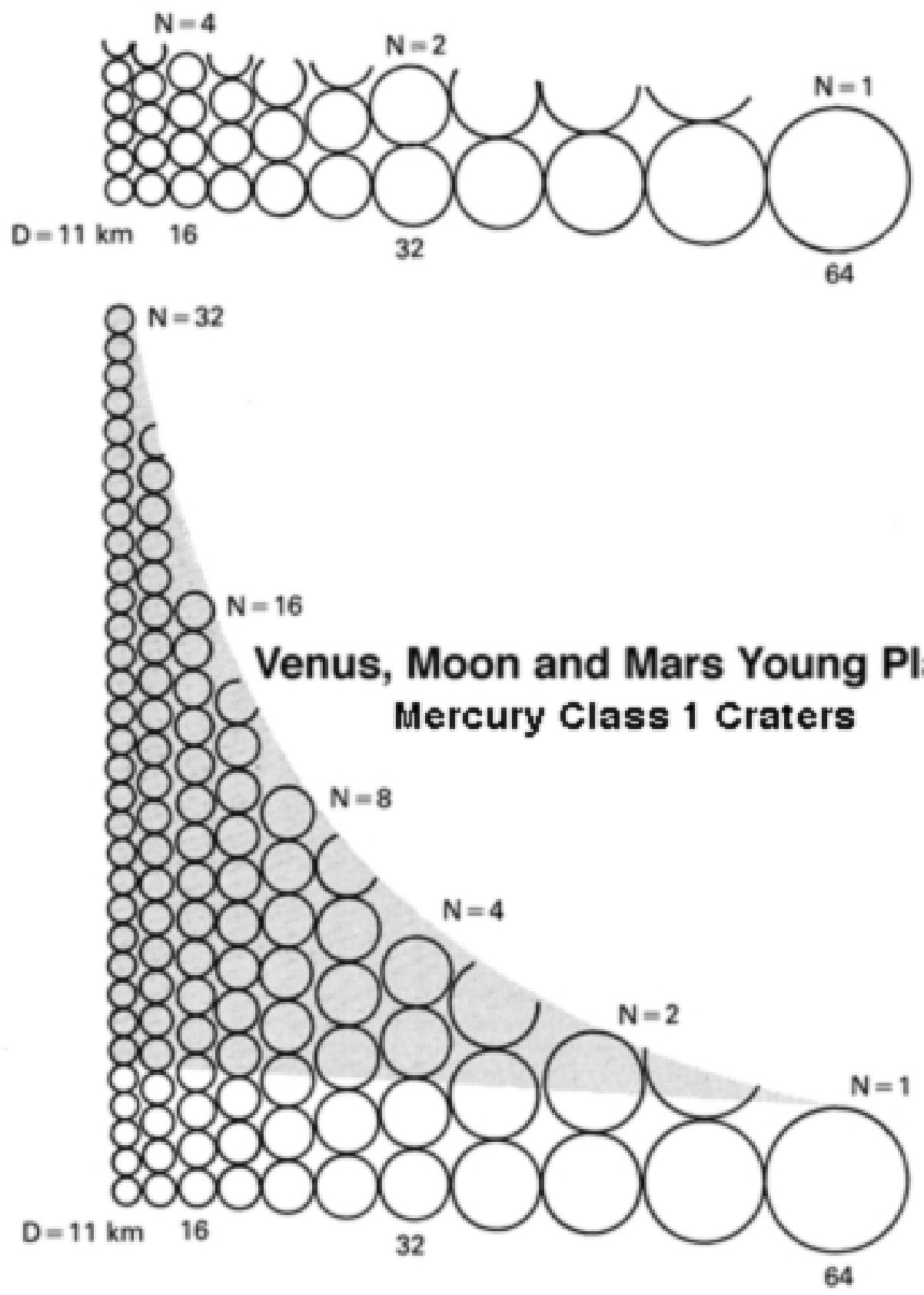

Fig. 2 This diagram graphically shows the difference between a differential -2 power law (top) that is characteristic of Population 1 at diameters less than about $50 \mathrm{~km}$ and a differential -3 power law characteristic of Population 2 (bottom). Each circle represents a crater of a given diameter. The shaded area in the bottom diagram is the difference between the two populations. 


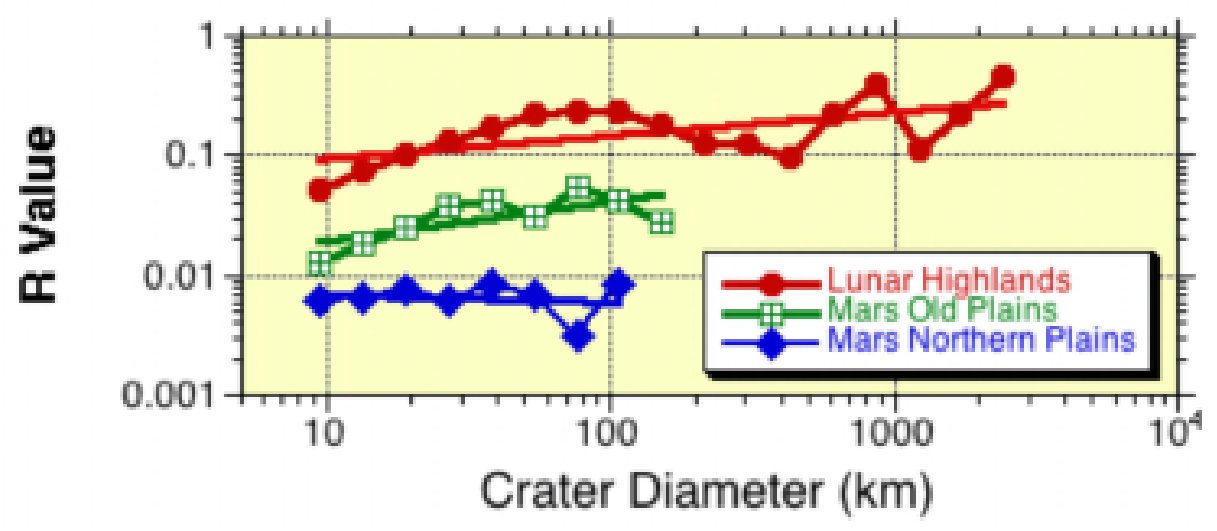

Fig. 3 Distorted $R$ plot where the "Crater Diameter" axis scale is much larger than the " $R$ Value" axis scale. The undistorted Lunar Highlands and Mars Northern Plains curves are shown in Figure 1 , and the undistorted Mars Old Plains is shown in Figure 9 , The solid lines are power law fits to the data.

As with any log-log plot, for the most effective visual communication of data, it is good practice to choose the horizontal (diameter) and vertical ( $\mathrm{R}$ value) scales of the plot axes to be the same, e.g., $R=0.01-$ 0.1 and $D=10-100 \mathrm{~km}$ should have equal lengths, otherwise the curves will appear distorted. To illustrate, we plot three different crater SFDs in Figure 3 using a longer $x$ axis than a $y$ axis. The figure has gross distortions that make the different populations (Population 1 and 2) look similar in shape to the fitted straight lines, thereby supressing statistically significant differences. We note that prior to the widespread use of computer software graphing programs, $\log$-log graph paper was used, which had equal $x$ and $y$ axes scales providing for undistorted plots. However, modern computer graphing programs allow to easily abandon that older paper-based convention, thereby enabling avoidable degradation of the best possible visual communication of data. Log-log plots created with these programs should preferably be adjusted to display them with equal vertical and horizontal scales.

\section{TERRESTRIAL PLANET CRATERING RECORD}

\subsection{Population 1 and Population 2}

\subsubsection{Earth}

The Earth is not very useful for reconstructing the impact cratering record due to its active geological history. The processes of plate tectonics, deposition and erosion have obliterated most of its cratering record. About $60 \%$ of the Earth's surface (the oceanic lithosphere) has been destroyed by seafloor spreading during the past 200 million years, or the last $4.5 \%$ of Earth history. The average ocean depth is about $5 \mathrm{~km}$, which would serve to screen out the smaller impacting objects, somewhat analogous to the screening of impactors by the Venus atmosphere (c.f. section 3.1.4). The continental areas also have been greatly modified 
by crustal deformation caused by plate tectonics, and by erosion and deposition. Only about 180 impact structures have been confirmed on the Earth. They range in size from $\sim 15$ meters to $\sim 300 \mathrm{~km}$ in diameter and have ages ranging from a few years to $2.4 \mathrm{Ga}$ (French, 1998). Almost all of these craters occur on continental craton areas. Consequently the crater statistics are not sufficient to characterize the SFD. There is no surface topographic crater record of the very ancient bombardment on Earth because the solid surface from that period has been almost completely renewed. However, there are indications of this impact history preserved in ancient zircon coatings (Trail et al. 2007), tungsten isotopes (Willbold et al. 2011), and layers of impact spherules caused by large impacts (Johnson \& Melosh, 2012); these are discussed further in section 4

\subsubsection{Moon}

The Moon is the best object in the inner Solar system to document the ancient cratering record. Unlike Mercury, Mars and Venus, the heavily cratered lunar highlands have not been greatly modified by internal or external activity and, therefore, preserve nearly the entire bombardment record better than any other terrestrial planet. The Moon's post-mare craters also separately record the geologically recent impacts.

Figure 4 shows the SFDs for four data sets of lunar craters: lunar highlands craters, fresh Class 1 craters, post-mare craters, and the Copernican and Eratosthenian craters. The lunar highlands, Class 1 and the postmare crater data are from the LPL Crater Catalog (Arthur et al., 1964); Copernican and Eratosthenian crater data are from a catalog by Wilhelms et al. (1978). Class 1 craters are defined as those having a very pristine morphology, a well-defined continuous ejecta blanket, and fresh secondary craters that post-date the surrounding terrain. They are some of the youngest craters on a planetary surface (Wood \& Anderson, 1978). The Copernican and Eratosthenian craters are defined by their stratigraphy; they are post-mare and they are also all Class 1 in their morphology. In Figure 4 we have plotted crater data only for $D>8 \mathrm{~km}$, although the source catalogs do have data at significantly smaller crater diameters. This lower diameter cut-off is made to avoid confusion with secondary craters; see Section 3.2 for discussion on this point. We also mention that a modern catalog of lunar craters of diameter $D \geq 20 \mathrm{~km}$, based on data from the Lunar Orbiter Laser Altimeter (Head et al. 2010), confirms the SFDs derived from the older imaging data.

As seen in the $R$ plots of Figure 4 , the lunar highlands have a high crater density, and a complex size frequency distribution; it is the model for the SFD characteristic of Population 1. The other three curves differ significantly from the highlands' curve: they have a much lower crater density and they have a nearly horizontal straight line shape in the $R$ plot, characteristic of Population 2. These represent the cratering that occurred during and/or after a rapidly declining period of the heavy bombardment. The freshest morphological Class 1 craters also have a size distribution the same as Population 2 craters, albeit with poorer statistics, as indicated by their larger error bars in Figure 4

The freshest large basin on the Moon is the $900 \mathrm{~km}$ diameter Orientale basin. It is thought to be the last basin formed by the LHB impactors, with an estimated age of about 3.8-3.7 Ga (c.f. Le Feuvre \& 


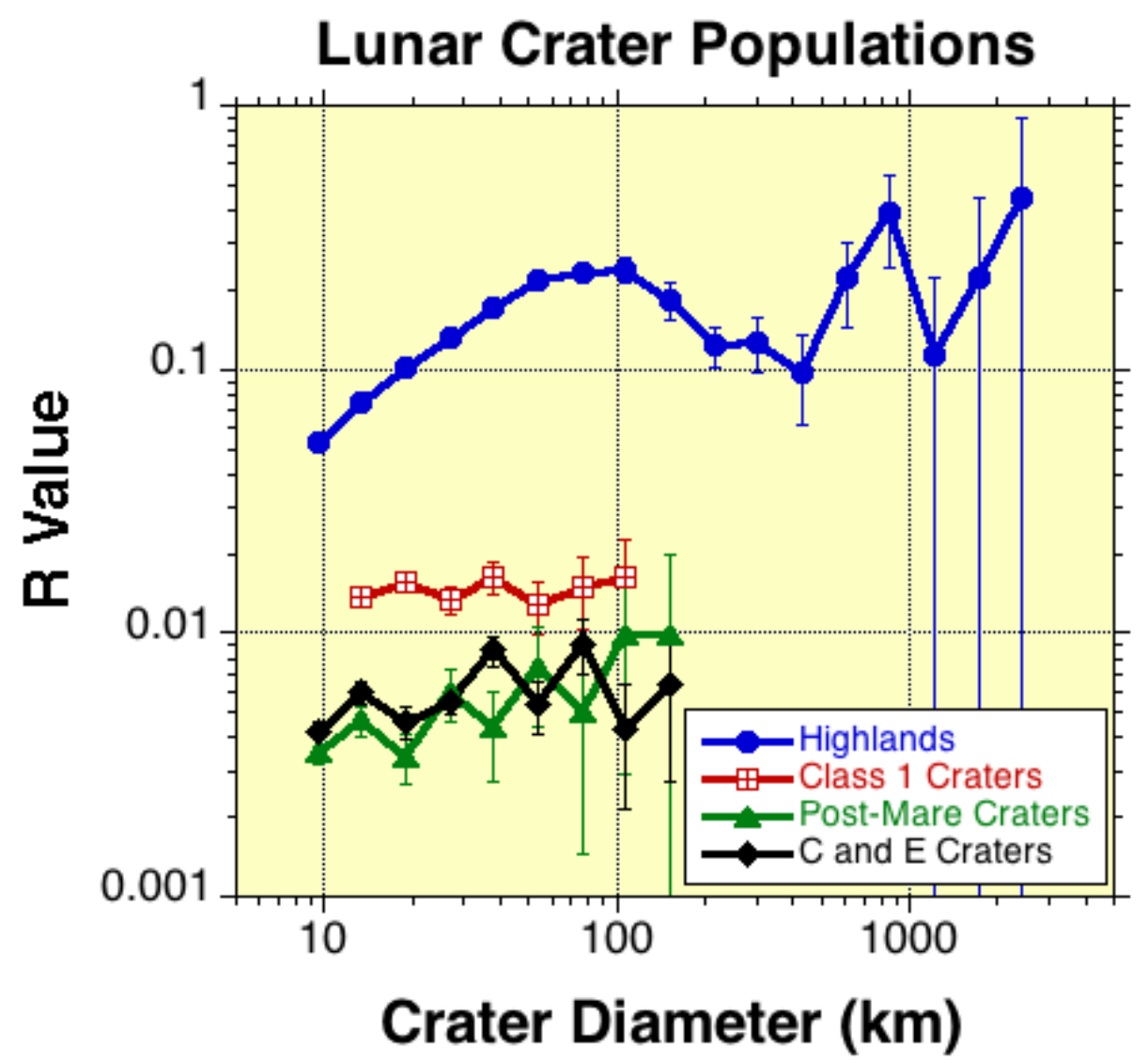

Fig. $4 R$ plot of the two crater populations on the Moon. The top curve (blue) is for the lunar highlands. The middle curve (red) is for all Class 1 lunar craters. The "Post-Mare Craters" (green) are only those craters that are superposed on the lunar maria, and the "C and E Craters" (black) are the Copernican and Eratosthenian craters identified stratigraphically as being emplaced during the Moon's youngest geological period; all of these are also Class 1 craters.

Wieczorek, 2011). Figure 5 shows the $R$ plot of newly-determined crater counts on the basin interior and the continuous ejecta blanket of Orientale; these can be called "post-Orientale" craters. (In the Appendix, Figure A-1 shows the imaged area of these counts.) For reference, the $R$ plot of the lunar highlands is also shown in this figure over the same diameter range. In the left panel is the post-Orientale curve; this curve slopes down to the left, but at a gentler slope than the lunar highlands curve. This is consistent with a mixture of Populations 1 and 2 (Strom et al., 2008). In the right panel of Figure 5, the post-mare craters from a proportional area have been subtracted from the post-Orientale curve to estimate the Population 1 fraction in the post-Orientale data. This yields a curve nearly parallel to that of the lunar highlands but lower by a factor of about 6.5 in the $R$ value. Because the Orientale basin may host more Population 2 craters than 


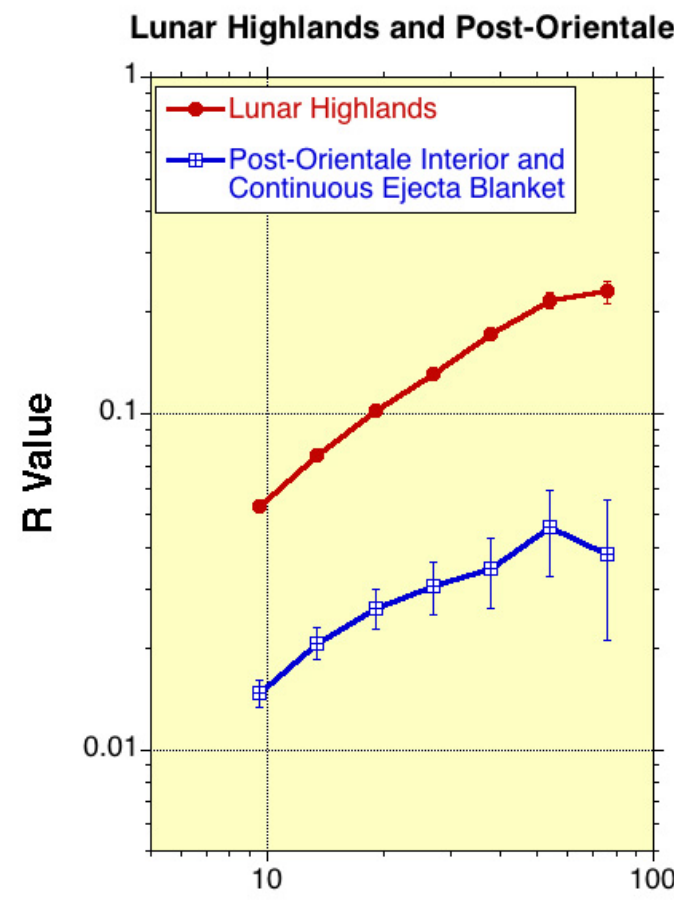

Crater Diameter (km)

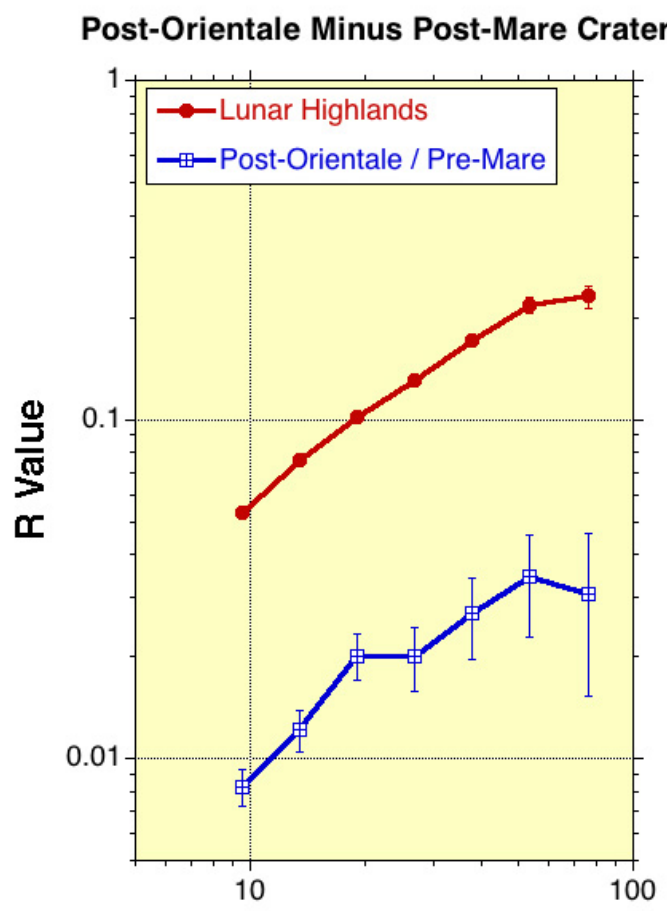

Crater Diameter (km)

Fig. $5 R$ plots of the post-Orientale craters (left) and the post-Orientale minus post-mare craters (right). The lunar highlands $R$ plot is shown in red for comparison.

indicated by just the post-mare crater density, we conclude that at the time of the Orientale basin formation, the Population 1 impact flux had decreased by a factor of at least 6.5 but still dominated over Population 2 .

Head et al. (2010) have also confirmed the presence of the two crater populations on the Moon in laser altimetry data obtained by the Lunar Reconnaissance Orbiter. They have shown that the post-mare crater population is different from the lunar highlands and that the Orientale basin has a crater population the same as the highlands, but at a lower crater density. This was also reported in earlier studies of Strom et al. (2005) and Marchi et al. (2009).

\subsubsection{Mercury}

Mercury has a heavily cratered surface with a widespread distribution of intercrater plains and a smaller area of relatively young smooth plains. Previous studies using Mariner 10 data recognized Population 1 (Strom, 1979), but the statistics of Population 2 were not good because of the relatively low image quality (i.e., low resolution and illumination conditions). However, images from the MESSENGER (MErcury Surface, Space ENviroment, GEochemistry, and Ranging) data have verified the existence of both Population 1 and Population 2 craters on Mercury. The largest areas of smooth plains on Mercury are the Caloris interior and 
exterior plains and the Northern Plains (Head et al. 2011). The MESSENGER mission has now provided the imaging data necessary to reconstruct more accurately the global cratering record, especially for the Population 2 craters. The MESSENGER orbital data is also used to count the freshest craters [morphological Class 1 from Arthur et al. (1964); Wood \& Anderson (1978)] on the heavily cratered equatorial areas on Mercury. These craters are the youngest craters on Mercury and consist of all rayed craters and those with pristine morphologies and well-developed ejecta deposits with superposed well-defined secondaries. The orbital data is also used for new counts on the Northern plains.

Figure 6 shows $R$ plots of the crater SFDs on the major geological units of Mercury. Collectively, the SFD for the heavily cratered terrains with interspersed intercrater plains (red curve) is similar in shape and magnitude to the lunar highlands shown in Figure 4. The upturn at diameters below $10 \mathrm{~km}$ is due to secondaries (Strom et al. 2011); this is discussed further in section 3.2. The green and blue curves are for the Caloris exterior plains and the Northern Plains. These relatively young plains are the same age (Head et al. 2011) and have a crater SFD intermediate between Population 1 and Population 2 indicating they are a mixture of the two populations, but dominated by Population 2 (Strom et al., 2008; Strom et al., 2011). Therefore, these plains formed at a time when the impact rate had fallen to a level where Population 2 was beginning to dominate, and well after the Caloris impact. The black curve is the $R$ plot of fresh Class 1 craters counted in the equatorial areas. These fall on an approximately horizontal line characteristic of "pure" Population 2.

The widespread intercrater plains of Mercury are older volcanic plains that are estimated to have been emplaced during the period of the LHB (Head et al., 2011, Strom et al. 2011). In Figure 7 we show the $R$ plots of two high crater density areas of Mercury, one with abundant intercrater plains (blue) and one with less abundant intercrater plains (green); for comparison, we also plot the lunar highlands (red). We see that the green curve has a shape and magnitude similar to those of the lunar highlands for crater diameters $D>25 \mathrm{~km}$, but at smaller diameters the crater density is lower than that of the lunar highlands; this corelates with the existence of intercrater plains near the margin of the high density crater area (Strom et al. 2011). We also see that the blue curve (for the area with abundant intercrater plains) has lower crater density at diameters all the way up to $100 \mathrm{~km}$. Many of the heavily cratered terrains on Mercury show this type of curve (Fassett et al. 2011). In a recent analysis, Marchi et al. (2013) have independently carried out crater counts of Mercury's heavily cratered terrain and reached similar conclusions; additionally they derived an age of the intercrater plains formation beginning at about $4 \mathrm{Ga}$.

In summary, the global crater counts from MESSENGER images show that Mercury has been impacted by both Population 1 and 2. Mercury's Population 1 record has been affected by the emplacement of intercrater plains while the largest areas of smooth plains (i.e., Caloris and Northern Plains) record a mixture of Population 1 and 2. 


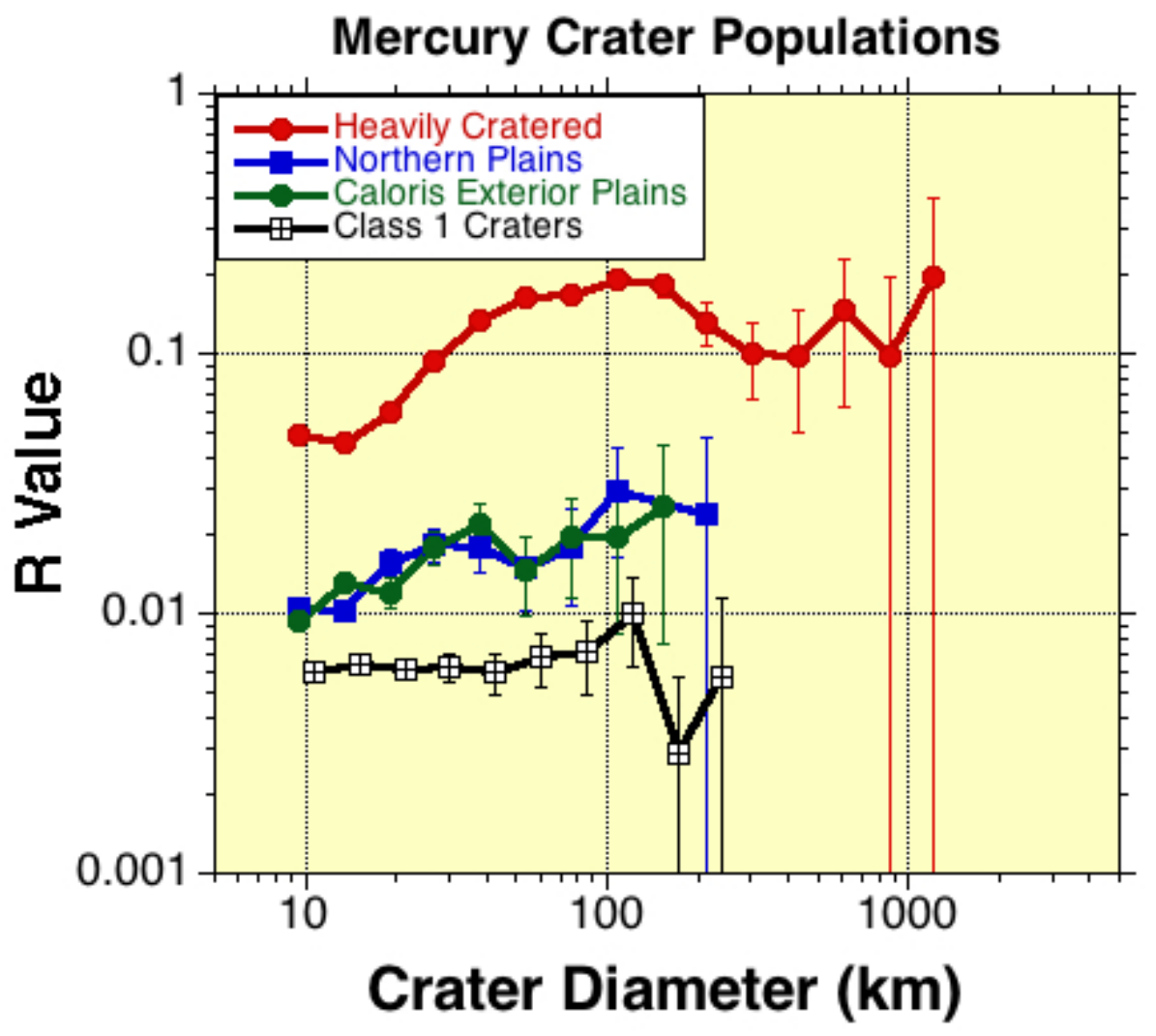

Fig. 6 The crater SFDs on several different terrains on Mercury: the heavily cratered terrains (red), the Northern Plains (blue), and the Caloris exterior plains (green); the black points are for all Class 1 craters in the equatorial regions.

\subsubsection{Venus}

Venus has undergone multiple global resurfacing events that have erased its ancient craters (Strom et al. 1994). Its thick atmosphere has progressively screened out smaller objects to severely modify the crater population below a diameter of about $25 \mathrm{~km}$. However, the largest craters and multiple craters (c.f. Strom et al., 1994) provide adequate statistics to reliably give important information on the geologically recent crater population.

Figure 8 shows the crater SFD on Venus; for comparison, we also show the $R$ plot for the Northern Plains of Mars. For crater diameters above about $25 \mathrm{~km}$, the crater density on Venus is almost an order of magnitude less than Northern Plains on Mars. Only very young craters are present on Venus because of multiple global resurfacing events (Strom et al., 1994). At diameters larger than $25 \mathrm{~km}$, the crater SFD has a -3 power law distribution akin to the lunar and martian young Population 2 shown in Figures 4 and 9 . At 


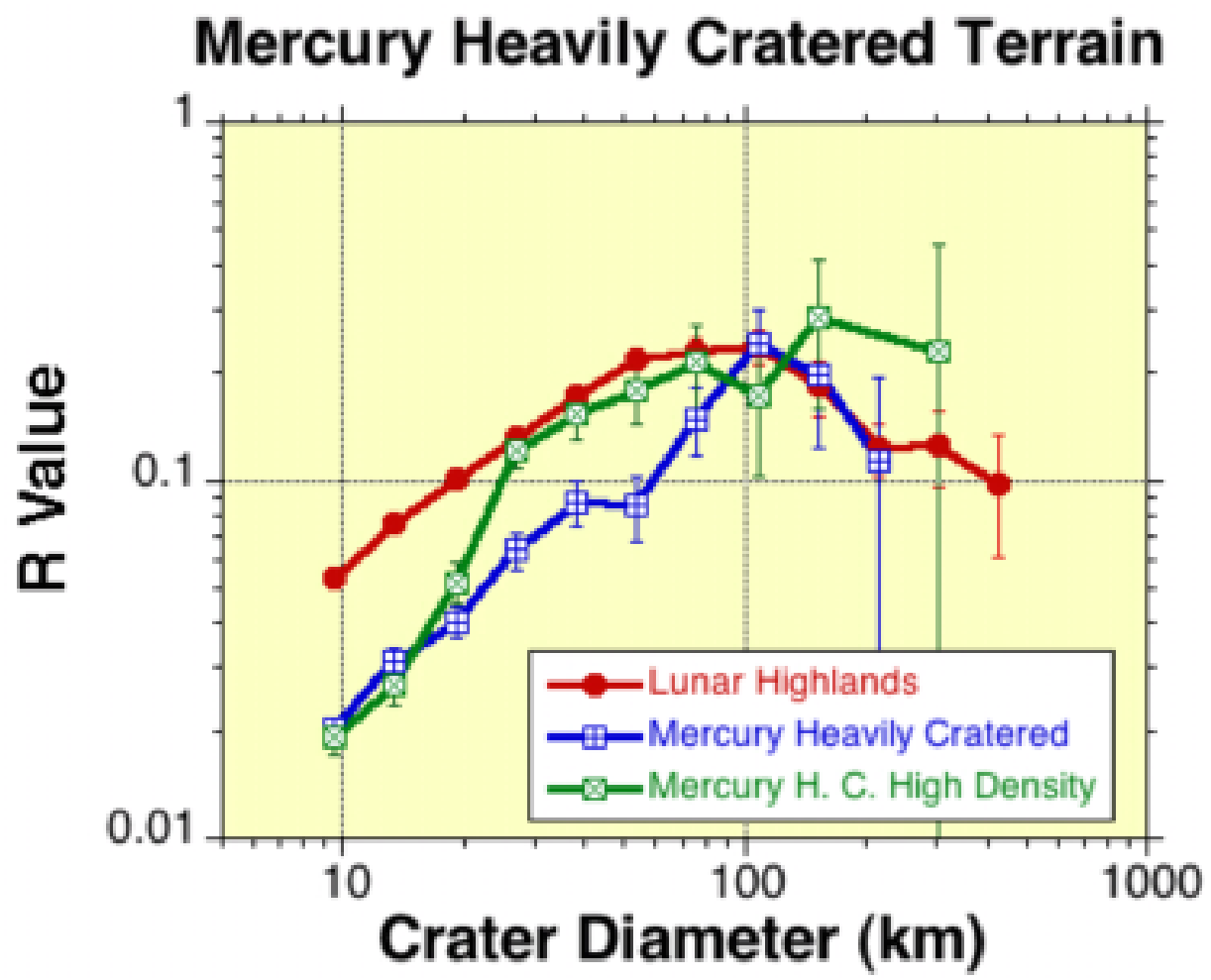

Fig. $7 R$ plots of the crater SFDs on Mercury's heavily cratered highlands in areas of relatively lower abundance of inter-crater plains (green points) and in areas of abundant inter-crater plains (blue points). For reference, the lunar highlands crater SFD is also shown (red points).

crater diameters below $25 \mathrm{~km}$, the curve sharply turns down. This is because impacting objects of small diameters are severely affected by atmospheric screening by Venus' thick 90-bar atmosphere (Zahnle, 1992). Part of the Venus crater population consists of clusters of craters (multiples) that result from fragmentation of the impacting object when entering the dense atmosphere. These comprise $16 \%$ of all Venus craters. Figure 8 shows the size distribution of multiples where the diameter is derived from the sum of the crater areas in the cluster. Multiples are probably formed by stronger, more consolidated objects that could resist atmospheric disintegration better than most other impacting objects, but still weak enough that they broke up in the atmosphere. The turnover of the curve for multiple craters does not occur until diameters less than $\sim 9 \mathrm{~km}$ (Figure 8). At larger diameters the curve is almost flat and consistent with a Population 2 distribution. This, together with the much lower crater density, strongly suggests that the impacting population on Venus was the same as Population 2 on the Moon and Mars. It is also strong evidence that the turnover of the crater curve is indeed due to atmospheric screening (Strom et al. 2005). 


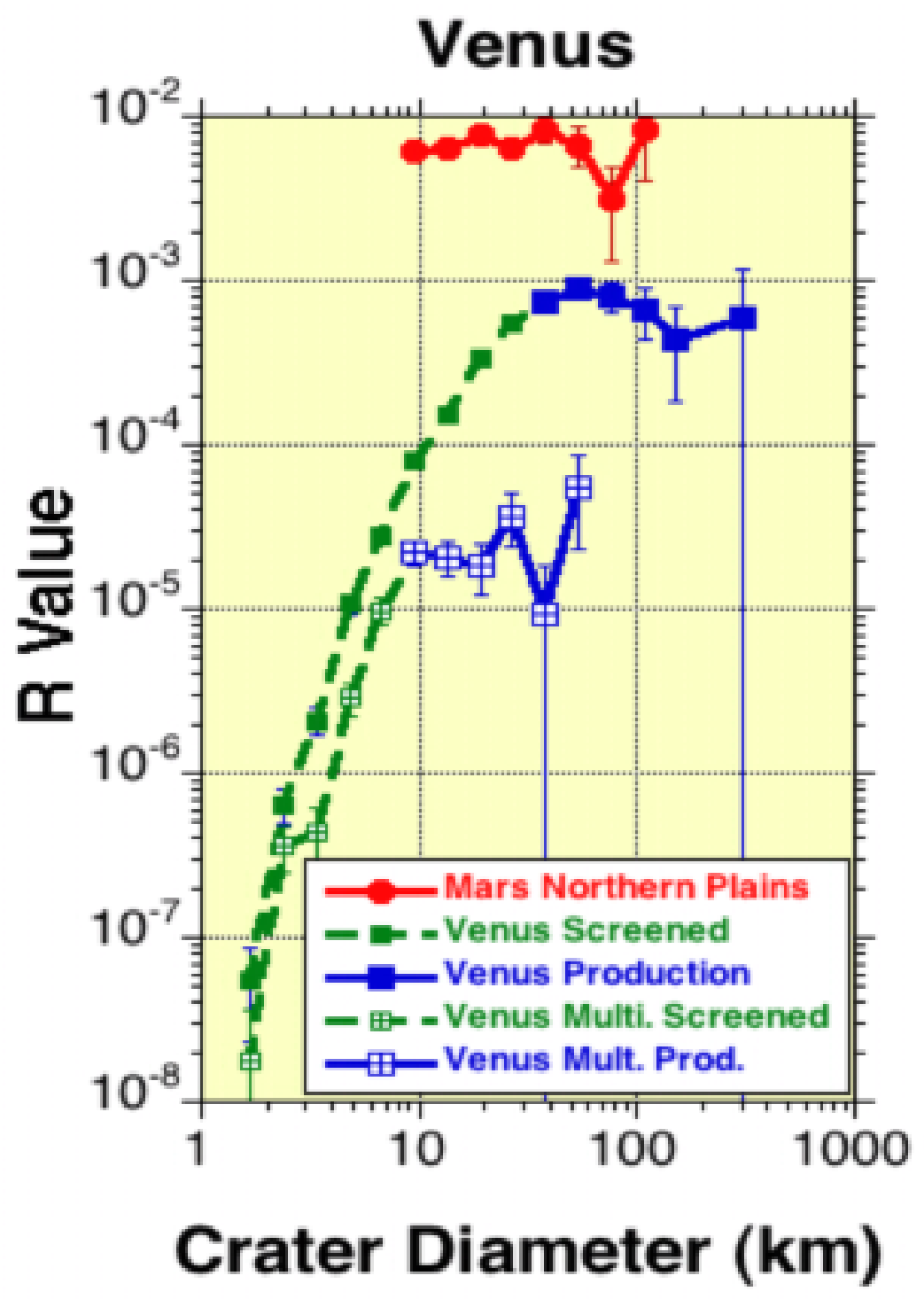

Fig. $8 R$ plots of the crater SFDs of all Venus craters and Venus' multiple craters (the two sets of blue points). The dashed lines (green) are the portions of the crater SFDs that are estimated to have been affected by screening in Venus' thick atmosphere; see text for explanation. For reference, we also show the $R$ plot of the Martian northern plains (red).

\subsubsection{Mars}

Mars provides an excellent record of the inner Solar system cratering history. It has numerous large areas with a wide variety of ages that provide good crater statistics in several geological periods spanning almost 
the entire history of the Solar system. Although some of the heavily cratered highlands have been greatly modified by internal processes and erosion and deposition, some portions of the relatively old areas show Population 1 very well, though at a lower crater density than the most heavily cratered areas. Furthermore, the large areas of younger plains show a considerable variety of ages that provide good counting statistics for determining the geologically recent cratering record. Figure 9 is a crater SFD of the different crater populations on Mars compared with the lunar highlands. (The various geologic units of Mars are as defined in the Geologic Map of Mars by Scott \& Carr (1978). In contrast with the lunar highlands, the Mars highlands curve shows a noticeable depletion of craters at diameters below about $30 \mathrm{~km}$. From the geological context, this is due to erosion and deposition. The Old Cratered Plains $R$ plot is for the surface unit east of Tharsis while the Hellas plains $R$ plot is for the surface unit within and surrounding the large Hellas impact basin. Both of these show the characteristic Population 1 shape albeit at lower density than the highlands. There are a number of young lightly cratered plains on Mars. Two representative examples, the northern plains and the Tharsis plains, are shown in Figure 9 Both have a horizontal straight line $R$ plot, characteristic of the Population 2 craters. In general, Mars displays the two crater populations and their transition better than other terrestrial planets because it has large surface areas with a variety of crater densities hence a variety of relative ages.

\subsection{Population S-the Secondary Crater Problem}

A distinct population of impact craters on planetary surfaces is caused by ejecta from primary impacts (e.g. Shoemaker, 1965, McEwen et al., 2005). The size and spatial distribution of secondary craters (Population S) generally depend on the size of the primary impact crater, the impact velocity, and the planet or satellite gravity field (e.g. Xiao et al. 2014). At small diameters, secondary craters outnumber primaries on terrestrial planets and the Moon by orders of magnitude because a single primary impact can produce thousands of secondary craters (e.g. Dundas \& McEwen, 2007). Large basins such as Orientale and Imbrium on the Moon have produced some secondaries up to $20 \mathrm{~km}$ in diameter. However, at such large diameters, their number is relatively small compared to primaries in heavily cratered terrain. Our $R$ plots for heavily cratered terrain have a lower diameter cut-off of $8 \mathrm{~km}$ to avoid confusion with the vast majority of basin secondaries.

Secondary impact craters are very widely distributed and dominate the small crater population on planetary surfaces. Robbins \& Hynek (2011) have shown that secondaries on Mars are very widespread and can affect crater age dating unless they can be unambiguously distinguished from primaries. Xiao \& Strom (2012) showed that secondaries dominate the small crater $(D<1 \mathrm{~km})$ population on both young and old lunar surfaces. The secondary craters' SFD is characterized by a -4 power law. Figure 10 shows three $R$ plots in which the dominant presence of secondary craters is evident in the sharp change of slope at small diameters. This upturn in the $R$ plots occurs at crater diameters below about $1 \mathrm{~km}$ on Mars. On the Moon secondaries can begin to affect the crater SFD at diameters less than about $1 \mathrm{~km}$, but on Mercury this occurs 


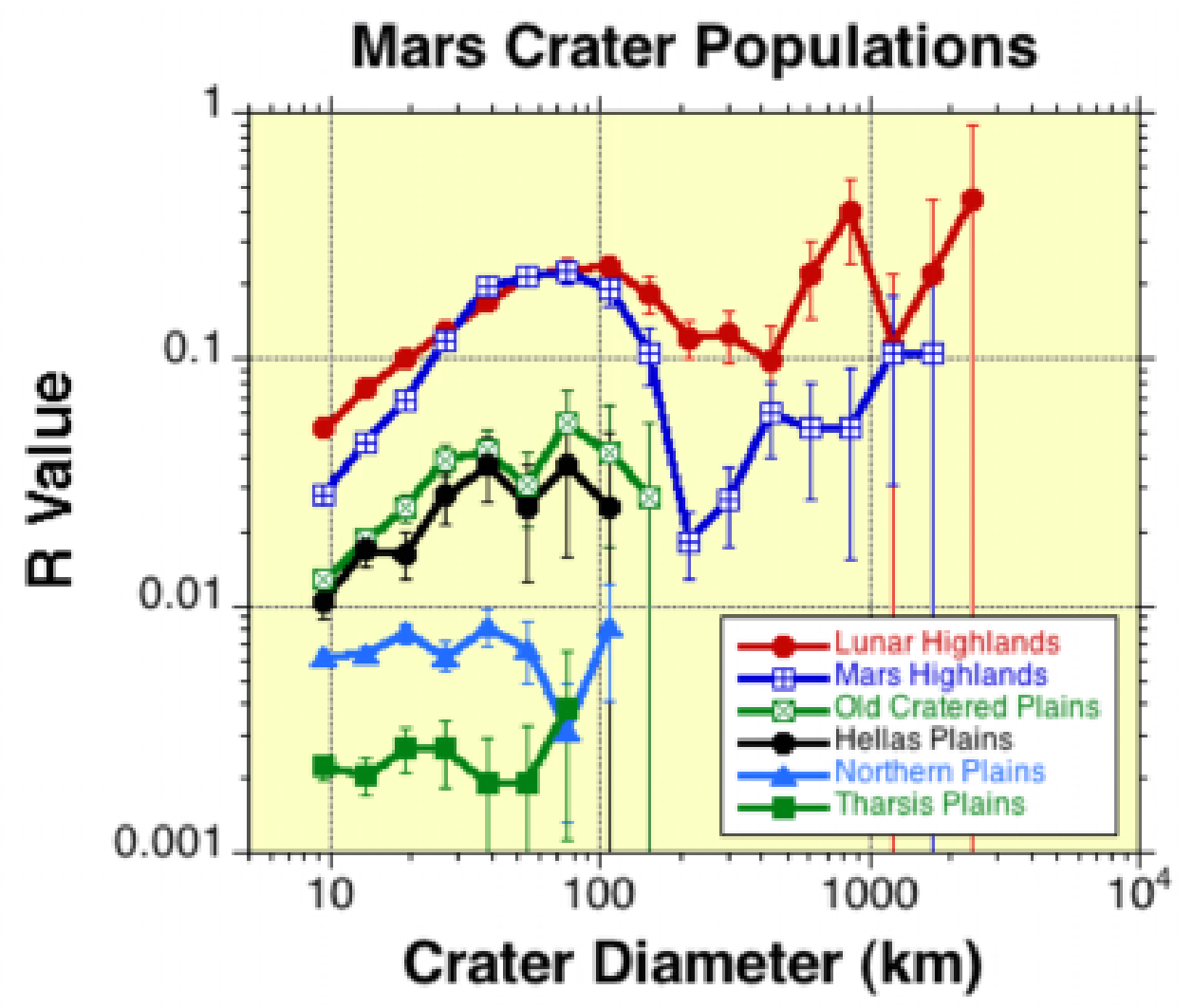

Fig. $9 R$ plots of the crater SFDs on several different terrains on Mars: the heavily cratered Mars highlands (dark blue), two moderately cratered areas - Old Cratered plains (green) and Hellas plains (black), two lightly cratered areas - the Martian Northern Plains (light blue) and the Tharsis plains (green). The Northern Plains are mainly the Vastitas Borealis region including geologic unit "Mottled Plains Unit", while the Tharsis plains are the region surrounding the Tharsis volcanic constructs including the geologic unit "Volcanic Plains on Tharsis Montes Region”. The Hellas Plains are the plains within the Hellas basin. The Old Cratered Plains are the plains east of Tharsis that partly includes the geologic unit "Old Volcanic Material".

at a diameter of about $10 \mathrm{~km}$. This is the main reason that most of the $R$ plots in this paper have a lower cut off diameter of $8 \mathrm{~km}$.

On the Moon, the basin secondaries mapped by Wilhelms et al. (1978) account for only $15 \%$ of the craters in the $8-11.3 \mathrm{~km}$ diameter bin, and even less at larger diameter bins. However, on Mercury the secondaries are larger than on any other terrestrial planet. The Mercury heavily cratered terrain as well as the Caloris exteroir plains $R$ plots have an upturn in the curve in the $8-11.3 \mathrm{~km}$ size bin that is due to secondaries as shown in Figures 10 and 6. The widespread distribution of larger secondaries on Mercury 


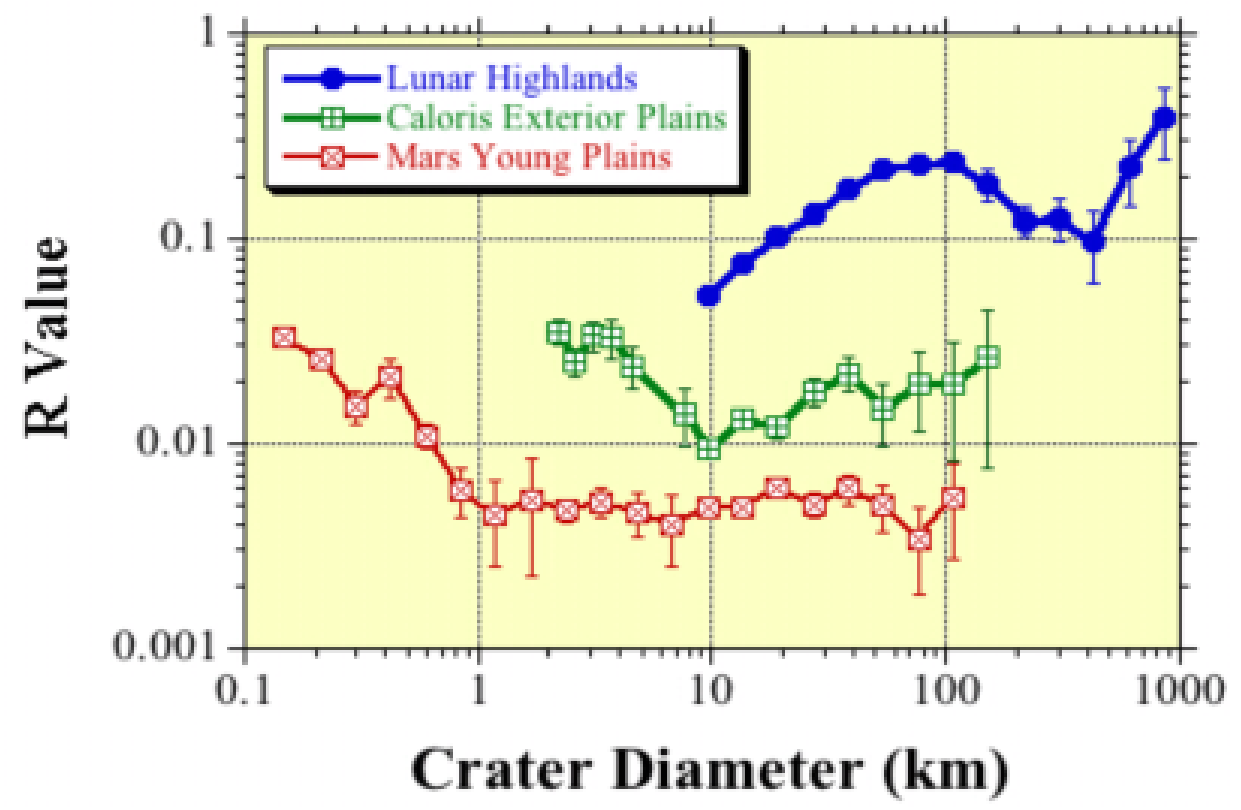

Fig. $10 R$ plots for the SFD on Mercury's Caloris Exterior Plains and Mars Young Plains, down to small crater sizes where secondary craters are abundant. On Mercury the upturn in the curve (green) at diameters below $10 \mathrm{~km}$ is dominantly secondary craters, while on Mars (red) the secondary upturn occurs below $1 \mathrm{~km}$.

may be due, at least in part, to a combination of higher secondary impact velocities, higher ejection angles and the larger surface gravity on Mercury (Strom et al. 2008; Strom et al., 2011; Xiao et al. 2014).

The upturn in the crater SFDs at small diameters has been interpreted by some authors to be the primary crater production function (e.g. Hartmann et al., 2007, Ivanov et al. 2002, Michael \& Neukum, 2010). This is very unlikely as demonstrated by McEwen \& Bierhaus (2006); Xiao \& Strom (2012). Direct evidence supporting this statement is found in the SFDs of craters with rays and bright halos on inner Solar system bodies which are most likely primaries. In Figure 11, we show the $R$ plots of Mars Young Plains craters down to small crater sizes, compared with two populations of rayed craters of small diameters, from about $1 \mathrm{~km}$ down to about 10 meters. The middle panel in the figure shows the $R$ plot of the small rayed crater population on Mars, and the "Lunar Bruno" curve in the bottom panel is for the bright haloed craters on the continuous ejecta blanket of the very young Giordano Bruno crater on the Moon. The Mars rayed craters show a gentle slope upward to the right in the two largest diameters but the statistics are poor and a -3 differential SFD is well within the error bars. The gentle decrease in the number of rayed craters at diameters less than $0.02 \mathrm{~km}$ is likely due to the loss of rays at decreasing crater size, analogous to the loss of multiple craters below $D \approx 8 \mathrm{~km}$ on Venus due atmospheric screening (cf. Figure 8). If there was 
a loss of rays throughout the diameter range counted then a systematic downward trend over the entire diameter range would occur, which is not present. This supports the interpretation that the upturn seen at small diameters, $D \lesssim 1 \mathrm{~km}$, in the $R$ plot of the Mars Young Plains craters (see Figure 10 ) as well as in other cratered terrains on Mars, is not reflecting the primary crater population, but is owed to large numbers of secondary craters in that size range. Likewise, the lunar Bruno crater curve (bottom panel of Figure 11) is flat in the $R$ plot down to diameters of $\sim 10$ meters, indicating that this small diameter primary crater population at geologically recent times on the Moon also shares the differential -3 slope characteristic of Population 2 craters at larger sizes.

Some crater counters believe they can distinguish between primaries and secondaries, but this is doubtful unless they have and use topographic data to determine their depth-to-diameter ratio. This technique is unlikely to be applied to every small crater due to their enormous numbers. Eliminating crater clusters or strings does not eliminate all secondaries because much ejecta are on high trajectories that produce randomly distributed craters, i.e., distant secondaries (Xiao \& Strom, 2012). These secondaries usually do not occur in clusters or chains, and may be highly circular in shape similar to same-sized primaries, making them difficult to distinguish from primaries. On Mercury, the contamination of secondaries might be extremely severe because some craters form very circular and isolated secondaries, even on continuous secondaries facies, probably due to the special target properties (Xiao et al. 2014). An empirical way to make the distinction between primaries and secondaries is to plot the crater counts on an $R$ plot. If the curve trends upward to the left at small diameters ( $D \lesssim 1 \mathrm{~km}$ for Mars and lunar craters, $D \lesssim 10 \mathrm{~km}$ for Mercury craters), then the count is likely contaminated with secondaries.

\subsection{Summary of the Inner Solar System Cratering Record}

Based on their different crater SFDs, the terrestrial planets and the Moon have been impacted by two populations of objects: Population 1 dominated at early times and was associated with a much higher impactor flux than Population 2 which dominated at later times but beginning at least as early as the formation of lunar maria and up to the present time. Figure 12 summarizes the two crater populations in the inner Solar system from the heavily cratered lunar highlands, the martian old cratered plains, and the younger more lightly cratered plains on the Moon, Mars, and Venus. Population 1 is responsible for the period of Late Heavy Bombardment, and Population 2 is responsible for the period mostly after heavy bombardment up to the present time. The Venus curve is a composite of the production population for all craters and for multiple craters only. The Mercury curve is for Class 1 craters, but Mercury's Northern Plains and the Caloris interior and exterior plains are mixtures of Populations 1 and 2 (see Figure 6). Also, large numbers of secondary craters dominate the small crater populations on these bodies; in the $R$ plots, the secondaries contamination is signalled by a distinct upturn for diameters $D \lesssim 1 \mathrm{~km}$ on the Moon and Mars, $D \lesssim 10 \mathrm{~km}$ on Mercury. 

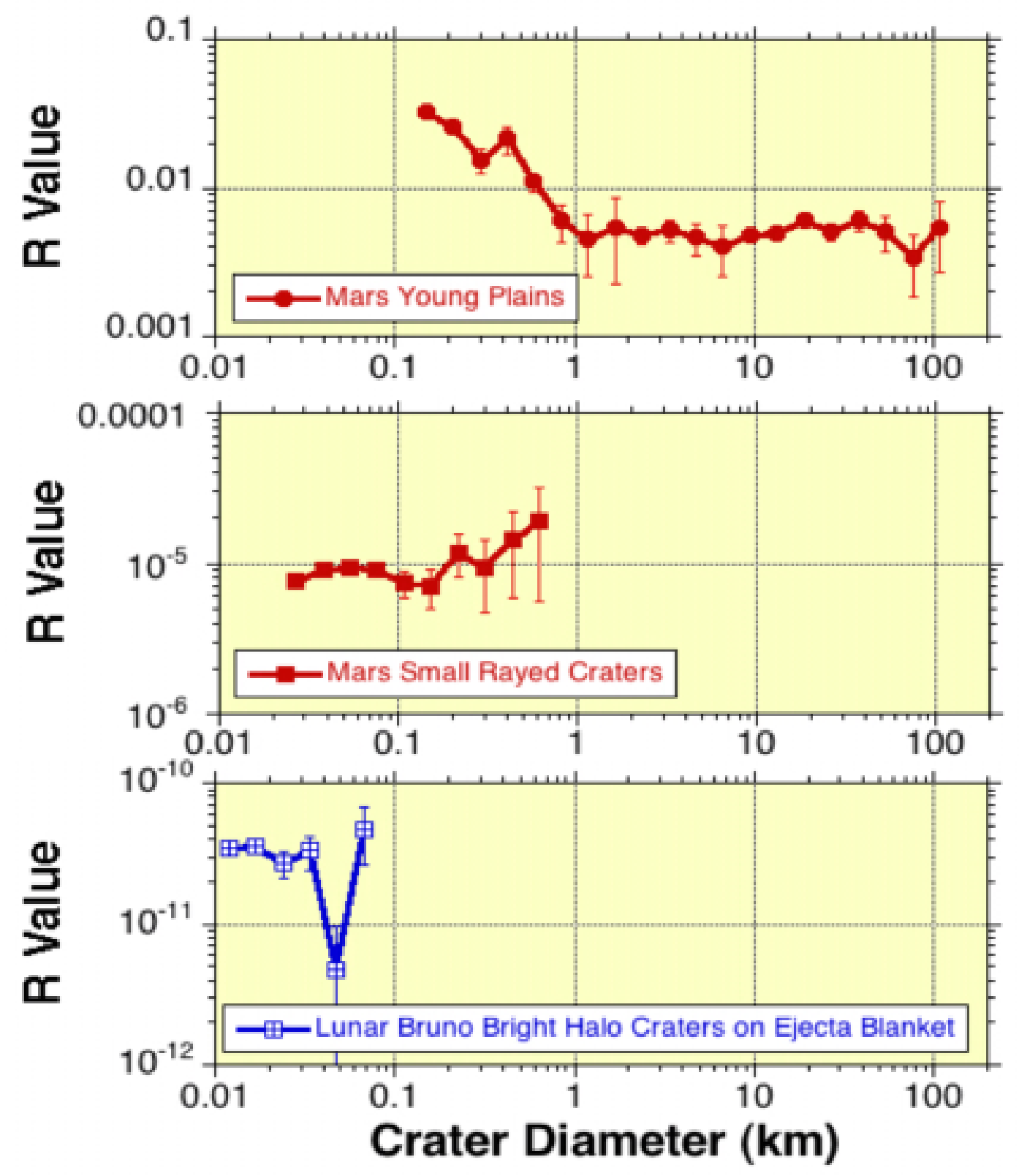

Fig. $11 R$ plots for the crater SFDs of Mars Young Plains down to sub-km diameter sizes (top panel), Martian small rayed craters (middle panel), and bright haloed craters on the continuous ejecta blanket of the young and very fresh Giordano Bruno crater on the Moon (bottom panel).

\subsection{The Outer Solar System Cratering Record}

The cratering record on outer solar system satellites appears to be very different from that in the inner solar system (Chapman \& McKinnon, 1986, Dones et al. 2009; Strom et al., 1981, 1990, McKinnon et al., 1991). Figure 13 is a group of $R$ plots of the SFD of impact craters on the satellites of Jupiter, Saturn, Uranus and 


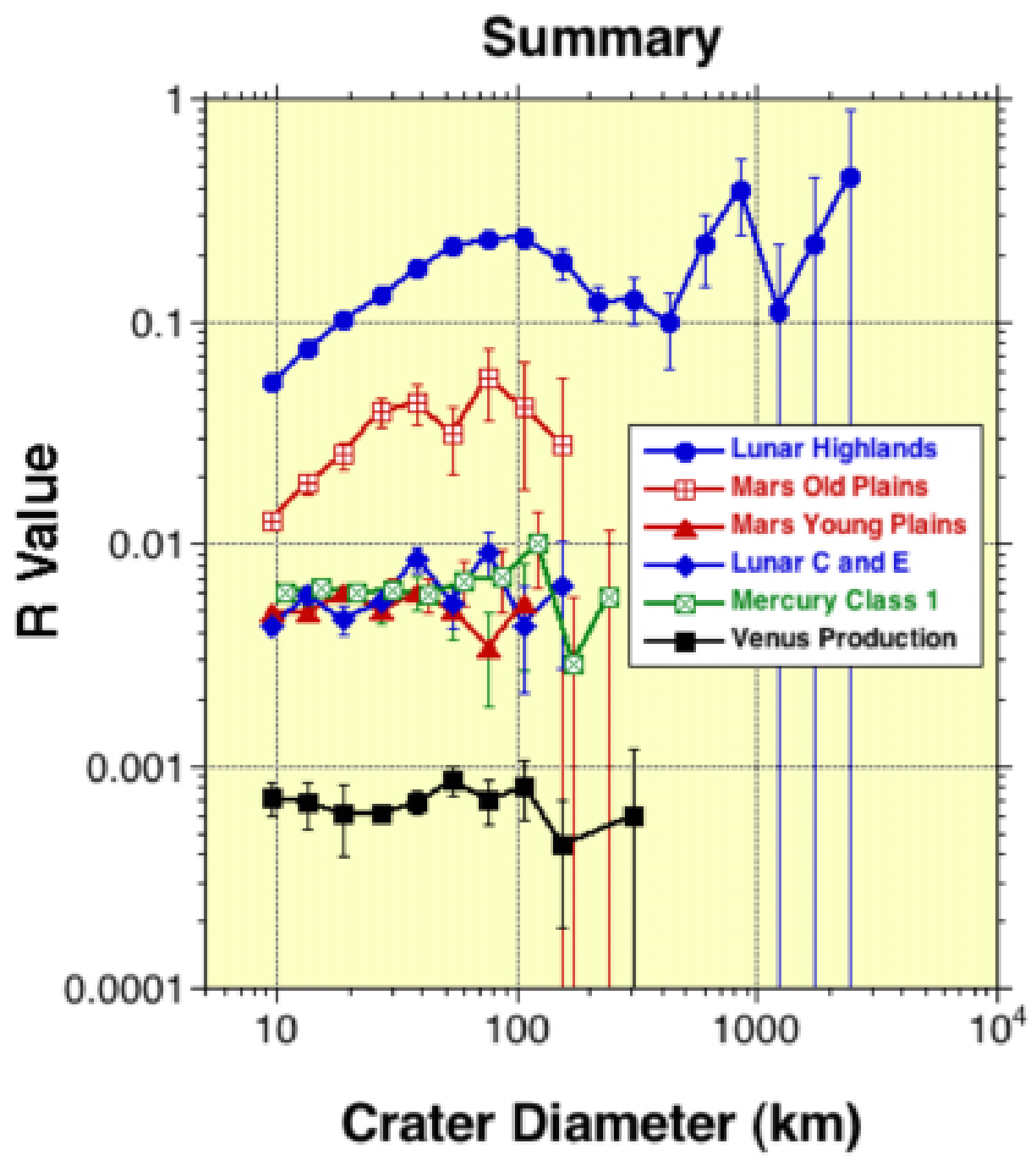

Fig. 12 These $R$ plots summarize the inner solar system cratering record for crater sizes in the range of about $10 \mathrm{~km}$ to about $1000 \mathrm{~km}$. They show two distinctly different crater populations. The curves above an $R$ value of about 0.01 have a complex shape characteristic of Population 1 , and the lower curves have a nearly horizontal straight line shape chacteristic of Population 2. The "Lunar C and E" craters are post-mare Copernican and Eratosthenian in age, and the "Venus Production" is a composite of the productions of all craters and multiple craters (see Figure 8).

Neptune compared to the lunar highlands. Only satellites with heavily cratered surfaces are shown in the plots. These data show that, with the possible exception of the heavily cratered surface of Miranda, the crater SFDs of the satellites are different from those of the lunar highlands Population 1 craters. On the Uranus 

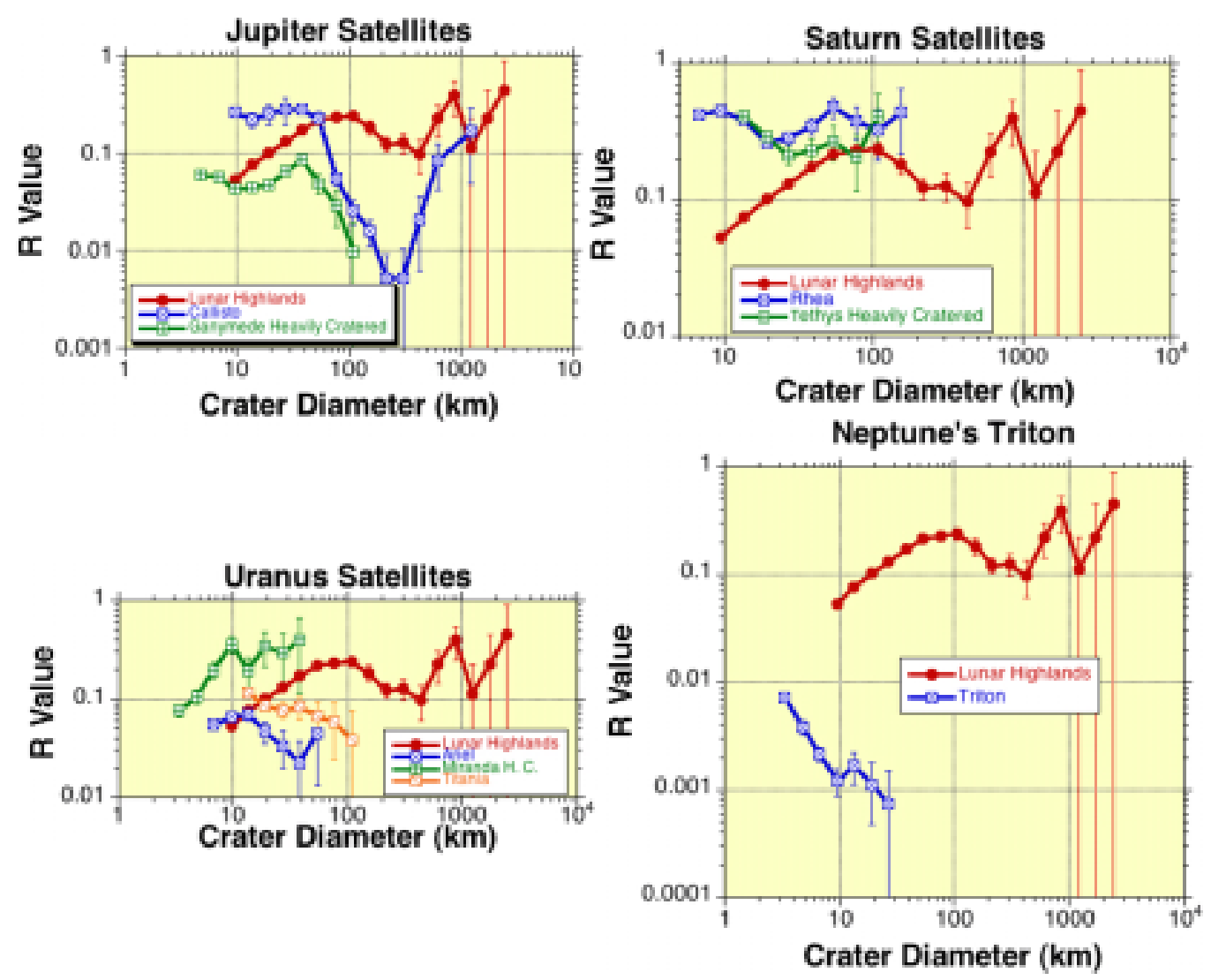

Fig. 13 The impact crater SFDs on the heavily cratered surface units of the outer planet satellites.

In each panel, for reference we also show the $R$ plot for the lunar highlands craters.

satellites Ariel and Titania, at smaller crater sizes the curves slope upward compared to the downward slope on the lunar highlands, but on the heavily cratered terrain on Miranda the curve slopes downward similar to the lunar highlands. On Triton the curve slopes upward at a steep angle as we go to smaller crater sizes. However, this satellite has been greatly resurfaced and these craters represent late impacts probably well after the period of late heavy bombardment. None of the outer planet satellites' crater populations resemble the cratering record on the heavily cratered terrain of the Moon and the inner planets. Therefore, Population 1 craters appear to be confined to the inner solar system. It is possible that the outer planet satellites may have been impacted by a mixture of projectile populations comprised of both comets and planetocentric objects. However, this topic requires further study and is beyond the scope of the present paper.

\section{DISCUSSION}

\subsection{Sources of Population 1 and Population 2 Impactors}

The size of an impact crater is related to the size of the impactor by the Pi-group crater scaling law (Croft) 1985; Schmidt \& Housen, 1987, Melosh, 1989; Collins et al., 2005). Strom et al. (2005) adopted this 
procedure to obtain the SFDs of the impactors responsible for the Population 1 and Population 2 craters. We follow the same procedure here; specifically, we use the web-based calculator of Melosh \& Beyer (1999) to compute the impactor sizes for each of the crater size bins. We assume a target type of "competent rock", adopt a common target and projectile density of $3000 \mathrm{~kg} \mathrm{~m}^{-3}$, a single impact angle of 45 degrees; we adopt surface gravity of $1.6 \mathrm{~m} \mathrm{~s}^{-2}$ and $3.7 \mathrm{~m} \mathrm{~s}^{-2}$ for the Moon and Mars, respectively. The lunar highlands craters were adopted as best representing Population 1 because the lunar highlands are the least affected by geological activity. The Martian young plains were adopted as best representing Population 2 for their better statistics compared with young crater populations on the other terrestrial bodies. For simplicity, a single value of the characteristic impact velocity was adopted for each of these $(18.9 \mathrm{~km} / \mathrm{s}$ for the Moon, and $12.4 \mathrm{~km} / \mathrm{s}$ for Mars). These values are the median impact velocities obtained in a recent self-consistent dynamical model of asteroid impacts on the terrestrial planets (Minton \& Malhotra, 2010). The resulting impactor SFDs are shown in Figure 14. Slightly different median impact velocities than adopted here can be found in the literature (e.g., Ivanov et al. (2002) obtain $17 \mathrm{~km} / \mathrm{s}$ for the median velocity of asteroid impacts on the Moon, Bottke et al. (2012) quote values as high as $21 \mathrm{~km} / \mathrm{s}$ for dynamical models of ancient asteroid bombardment on the Moon). If we adopt impact velocities that are smaller or larger by $20 \%$ than the nominal value adopted here (i.e., $15.1 \mathrm{~km} / \mathrm{s}$ or $22.7 \mathrm{~km} / \mathrm{s}$ ), the peak of the lunar highlands impactors' curve shifts to $\sim 0.5 \mathrm{~km}$ larger or smaller impactor diameter, respectively, but the shape of the distribution does not change.

Also plotted in Figure 14 is the available data on the size distributions of the near Earth objects (NEOs) and the main belt asteroids (MBAs). The NEO size distribution is based on the bias-corrected LINEAR diameters for NEOs (Stuart \& Binzel, 2004). The MBAs size distribution is based on four published data sets: 1) Spacewatch (Jedicke \& Metcalfe, 1998); 2) Sloan Digital Sky Survey (SDSS; Ivezić et al. (2001)); 3) Wide-field Infrared Survey Explorer (WISE) first release data (Masiero et al., 2011) and 4) Subaru Main Belt Asteroid Survey (Yoshida et al. 2003). From the SDSS data set, we used the "red" asteroids data, and from the other data sets we used only the data on the inner part of the main asteroid belt.

The Spacewatch survey did not measure albedos nor determine taxonomic types. In order to convert their asteroid absolute magnitude data to asteroid diameters, we used weighted average albedo values that were calculated as follows. (a) We used the Spacewatch dataset of absolute magnitude $(H)$ from Table IV of Jedicke \& Metcalfe (1998, p. 256). This table partitions the main belt into three parts, inner, middle, and outer, and gives binned statistics for the numbers of asteroids in 0.5 magnitude-wide bins. (b) We used the results of Yoshida et al. (2003) for the statistical ratio of the relative abundance of different taxonomic types (S- or C-type asteroids) in each part of the main belt. Yoshida et al. (2003) carried out a deep color survey of the main asteroid belt using the Subaru telescope; in this work, they obtained the colors and approximate orbital locations (inner/middle/outer belt) of $861 \mathrm{MBAs}$, and estimated the relative abundance ratios of $\mathrm{S}$ - and C-types in the inner/middle/outer main belt. (c) We calculated the average albedo values, $\langle A\rangle$, for each taxonomic type from the catalog of asteroid albedo and taxonomic types in the Planetary Data System 
catalog 11 We found $\langle A\rangle=0.21$ for S-type, and $\langle A\rangle=0.05$ for C-type. These values are consistent with recent estimates from infrared space surveys such as WISE and AKARI (Masiero et al., 2011; Usui et al., 2013). (d) Finally, we calculated a weighted average albedo for each partition (inner/middle/outer) of the asteroid belt. For example, for the inner MBAs, Yoshida found 233 S-type and $112 \mathrm{C}$-types, so the weighted average albedo for inner belt MBAs is $(0.21 \times 233+0.05 \times 112) /(233+112)=0.158$.

In Figure 14 the derived impactor SFDs of Population 1 and Population 2, as well as the data for the MBAs and NEOs are shown in an $R$ plot. The vertical position of the asteroid curves is arbitrary; these positions were chosen to clearly compare the shapes of the various curves. This figure differs slightly from a similar one published in Strom et al. (2005) in that the larger lunar basins have been included in the Population 1 curve and we have also included the WISE data for MBAs. Although the impact mechanics for large basins are not as well understood as for smaller craters (and old lunar basins may have formed in a hot crust, which may also affect the final crater size), the size bins at these diameters are so large (several hundred $\mathrm{km}$ ) that the uncertainty in the derived projectile mean diameter is probably within the size bin.

We see that the Population 1 impactor curve is very well matched with the curve for the MBAs. Only the largest size bin representing the three largest lunar basins (Imbrium, South Pole-Aitken, and Procellarum) is significantly below the MBAs data point. This may be due to the unrecognized structure of other large basins that formed early in the period of LHB and were obliterated by the heavy bombardment. Feldman et al. (2002) have found geochemical signatures of large basins on the Moon's far side that are similar to that of the Procellarum basin. These are at least part of the missing basins.

There are two conclusions that can be drawn from the impactors/asteroids comparison in Figure 14 First, the size distribution of MBAs is virtually identical to the size distribution of Population 1 impactors; this was also observed by Neukum et al. (2001). This result indicates that the Population 1 impactors originated from Main Belt Asteroids or possibly a population that had the same size-distribution as the contemporary inner main asteroid belt. Second, the comparison of LINEAR data and the young crater Population 2 strongly indicates that Population 2 craters were made by impactors derived primarily from Near Earth Objects. Supporting evidence that inner Solar system impactors were asteroids rather than comets is found in trace element analyses of lunar samples returned during the Apollo program (Kring \& Cohen, 2002). Furthermore, direct fragments of impactors have been identified in a recent study of ancient ( $>3.4 \mathrm{Ga})$ and younger $(<3.4 \mathrm{Ga})$ lunar regolith samples; these show that lunar impactors were primitive chondritic asteroids prior to $\sim 3.4 \mathrm{Ga}$, but the younger impactors have more diverse chemical compositions (Joy et al. 2012).

Let us consider in some detail the case of Population 1 impactors. Many previous studies have held that the ancient craters were made by a declining population of planetesimals in the inner Solar system that were left-over from planet formation. However, such a source is untenable because the typical dynamical lifetimes of planetesimals in planet-crossing orbits in the inner Solar system are $<10^{7}$ years Gladman 
et al. 1997, Ito \& Malhotra, 2006). When collisions are taken into account, the lifetime of an inner Solar system left-over planetesimal population is reduced even further (Bottke et al. 2007). The only known long-lived population that is a viable source of the Population 1 impactors is the main asteroid belt (but see discussion below about the Hungaria asteroids). A main asteroid belt source is consistent with the close match between the old Population 1 impactors and the contemporary MBAs, provided that (a) the shape of the MBAs' size-frequency distribution achieved a steady-state at least as early as $\sim 4 \mathrm{Ga}$, and has remained nearly unchanged since then, and (b) a dynamical mechanism existed at ancient times for transporting main belt asteroids into planet crossing orbits in a size-independent way. We discuss the latter condition in detail in Section 4.3. For the former condition, we note that numerical modeling studies by Cheng (2004) and Bottke et al. (2005) of the collisional evolution of the asteroid belt find that its size distribution changes little after the first $\sim 100 \mathrm{Myr}$.

There is additional evidence in the crater record supporting the hypothesis that the main asteroid belt was the primary source of the Population 1 impactors. This evidence lies in a comparison of the crater size distributions of the heavily cratered terrain of Mars, the Moon, and Mercury at the larger diameters where the curves have significant downturns to steeper slopes (i.e., more negative power law index; Figure 15. The downturn in the Mercury crater curve occurs at a larger diameter size-bin than on the Moon, whereas on Mars, the downturn occurs at a smaller diameter size-bin, as indicated by the arrows in Figure 15. We estimated the location of the peak of each of the $R$ plots in Figure 15 by fitting a 5th order polynomial to each curve; the peaks in the best-fit polynomials occur at crater diameters of $99_{-5}^{+7} \mathrm{~km}, 81_{-3}^{+5} \mathrm{~km}$ and $69_{-5}^{+1}$ $\mathrm{km}$ for Mercury, Moon and Mars highlands, respectively. This systematic shift from Mars to the Moon to Mercury of the "downturn diameter" of large craters is consistent with an origin of impactors from the Main Asteroid Belt, because the median impact velocities of these asteroids are higher on Mercury and lower on Mars, compared to the Moon. Use of the Pi-group scaling law and adopting the median impact velocity of MBAs for each planet $(38.1 \mathrm{~km} / \mathrm{s}, 18.9 \mathrm{~km} / \mathrm{s}$ and $12.4 \mathrm{~km} / \mathrm{s}$ for Mercury, Earth-Moon, and Mars respectively (Minton \& Malhotra 2010), yields corresponding peak impactor sizes of $3.1_{-0.2}^{+0.2}, 3.4_{-0.1}^{+0.3}$ and $3.9_{-0.3}^{+0.05} \mathrm{~km}$, respectively. Within the $\pm 0.5 \mathrm{~km}$ uncertainty owed to the uncertainty in the mean impact velocities, these peak diameters of the Population 1 impactors on Mercury, Moon and Mars are the same. They are also the same as the local peak (near $D \sim 3-4 \mathrm{~km}$ ) of the $R$ plot of the MBAs size distribution (cf. Figure 14). In other words, for the same impactor size distribution, the systematic differences in the mean impact velocity at Mercury, Earth-Moon and Mars, produce a shift in the crater sizes that are consistent with the observed shifts in the crater size distributions of Population 1 on Mercury, Moon and Mars. These shifts are therefore consistent with the hypothesis that the objects responsible for the Population 1 craters originated directly in the Main Asteroid Belt. This shift and its implication for the orbits of the impacting objects were first noticed by Strom \& Neukum (1988); its significance and connection with the Late Heavy Bombardment was explained by Malhotra \& Strom (2011). 
This shift also indicates that Mercury Population 1 was unlikely to have been due to Vulcanoids interior to Mercury's orbit (Stern \& Durda, 2000) because Vulcanoids would have impacted Mercury at about 13-14 $\mathrm{km} / \mathrm{s}$, similar to the impact velocity of asteroids at Mars. In this case the curve would show an offset similar to that of Mars; this is not observed. Also, if Vulcanoids existed, they may not be the main impactor-source for the Population 2 craters on Mercury, unless they had the same SFD as the NEOs. The MESSENGER spacecraft has not yet discovered any candidate Vulcanoids, indicating this hypothesized asteroid belt may have been depleted if it once existed.

On the other hand, with regard to the Population 2 craters, Figure 14 shows that the SFD of projectiles responsible for these is quite different from that of the Population 1 impactors. The differences are illustrated by the value of the asymptotic slope of the power law SFD at small diameters, $D<2 \mathrm{~km}$ : the Population 2 impactors have $\mathrm{a}-2.8$ asymptotic slope that is significantly steeper than the Population 1 impactors' -2.2 slope. Moreover, Figure 14 also shows that the SFD of the Population 2 impactors is very similar to that of the NEOs. This is perhaps the most direct evidence that the source of the Population 2 impactors is the NEOs. However, this conclusion raises a number of issues that we discuss below.

The NEOs are a transient population, with typical dynamical lifetimes $\sim 10^{7}$ years whereas Population 2 craters have accumulated over more than $\sim 3$ gigayears. Indeed Le Feuvre \& Wieczorek (2011) show that the density of Population 2 craters on the Moon is consistent with a nearly constant impact flux similar to that of the contemporary NEO impact flux over the past $\sim 3.5$ gigayears; Grieve \& Shoemaker (1994) and Neukum \& Ivanov (1994) had previously reached a similar conclusior How can this be reconciled with the short dynamical lifetimes of NEOs? Quite independent of the recently discovered similarity of the Population 2 craters and the NEOs' size distributions, previous dynamical studies of asteroids have indicated that the transient population of NEOs can be maintained in nearly steady state over gigayear timescales by being resupplied primarily from the main asteroid belt [see review by Morbidelli et al. (2002)]. Importantly, the steeper size distribution of NEOs compared to that of the main asteroid belt indicates that the dynamical transport process must be size-dependent, favoring the injection of smaller asteroids into the inner Solar system. A number of studies have argued that the key ingredient is the effect of non-gravitational forces owed to thermal radiation, specifically the Yarkovsky and YORP effects; Bottke et al. (2006) provide a recent review.

The Yarkovsky effect, named for the Polish engineer who discovered it more than a century ago, is a small thermal thrust that is produced when small airless spinning bodies orbiting the Sun emit thermal radiation in equilibrium with absorbed sunlight but with a small delay owed to thermal inertia; this small thrust causes a net secular orbital drift that depends on the size and spin and material properties of the body. The same physical process also produces a torque that modifies the small body's spin rate and spin axis

2 It is possible that the flux has varied by a factor of two or three over the past $\sim 3$ Gyr Hartmann et al. 2007 Marchi et al. 2009. Kirchoff et al. 2013). The crater record that we are examining is integrated over any variations in the flux that may have occurred and does not affect the conclusion that Population 2 is from NEOs. 
orientation, and is referred to as the YORP (Yarkovsky-O'Keefe-Radzievskii-Paddack) effect (Rubincam. 1988). Farinella \& Vokrouhlicky (1999) showed that over a few tens of millions of years these effects are large enough to push a significant number of sub-20-km size asteroids into strong Jovian resonances; the latter then deliver them into terrestrial planet-crossing orbits and thereby into the NEO population. The Yarkovsky effect and the YORP effect are most significant for objects between $10 \mathrm{~cm}$ and $10 \mathrm{~km}$ diameter; both effects diminish significantly beyond this size range. Morbidelli \& Vokrouhlický (2003) numerically modeled the dynamical origin of NEOs from MBAs, finding that, under a plausible range of adopted model parameters, the combination of collisions and the Yarkovsky and YORP effects roughly explains the steeper size distribution of the NEOs compared with the SFD of their source, the main asteroid belt. Further detailed studies are needed to determine whether the difference between the size distribution of the NEOs and MBAs is quantitatively fully accounted for by these non-gravitational effects, or whether this difference hides additional surprises.

Regardless of the reasons for the difference between the size distributions of the NEOs and of the main asteroid belt, the conclusion that Population 2 impactors' size distribution is similar to that of the NEOs holds.

It is of some interest to note that recent studies of the spatial distribution of young craters on the lunar surface find a significant longitudinal asymmetry due to the Moon's synchronous rotation (Morota \& Furumoto, 2003). The magnitude of this asymmetry is roughly consistent with the NEOs being the impactors (Gallant et al. 2009, Ito \& Malhotra, 2010, Le Feuvre \& Wieczorek, 2011). However, Ito \& Malhotra (2010) note a small discrepancy between the observations and the theoretical model and suggest that it may indicate a missing tail of low velocity Earth-Moon impactors JeongAhn \& Malhotra (2010).

\subsection{Age and Duration of the Late Heavy Bombardment}

The existence and properties of the two crater populations support the hypothesis of a "terminal lunar cataclysm", and, more widely, that the Late Heavy Bombardment (LHB) was a spike in the impact flux common to all the terrestrial planets, and that the spike consisted of bombardment by Population 1 projectiles whereas the post-spike projectiles have been Population 2. However, these impact crater data on their own do not constrain the timing and duration of the LHB. The latter are obtained from laboratory analysis of lunar samples and meteorites.

The analyses of samples from the Apollo lunar program showed that the lunar crust is $\sim 4.5$ Gyr old (Tera et al. 1973, Norman et al., 2003), but that several hundred million years subsequent to differentiation and crust formation, the lunar highlands suffered extensive mobilization of $\mathrm{Pb}$ isotopes and widespread impact metamorphism over a relatively short time interval ( $\sim 200$ myr) that ended $\sim 3.8 \mathrm{Ga}$ (Turner et al. 1973: Tera et al., 1974). This first led to the "lunar cataclysm" hypothesis of a spike in the bombardment at $\sim 3.9 \mathrm{Ga}$. 
The ages of the large lunar basins also apparently cluster near 3.9 Ga (Turner et al., 1973, Ryder, 2002). Also, the Martian meteorite Allan Hills 84001 records a shock event at $3.92 \mathrm{Ga}$ (Turner et al., 1997). These disparate pieces of evidence suggest a spike in the impact flux in the inner Solar system several hundred million years after the formation of the planets.

On Earth, there is possible evidence of the LHB recorded by impact generated metamorphic overgrowths on zircons older than $\sim 3.5 \mathrm{Ga}$. The ages of the over-growths cluster at $\sim 3.9 \mathrm{Ga}$ and may be due to multiple impact events associated with the LHB (Trail et al., 2007). Willbold et al. (2011) report that analysis of $\sim 3.8$ billion-year-old rocks from Isua, Greenland revealed a significantly higher isotopic tungsten ratio ${ }^{182} W /{ }^{184} W$ than modern terrestrial samples; they suggest that the Late Heavy Bombardment may have triggered the onset of the current style of mantle convection on the Earth. However, interpretation of the terrestrial Hadean eon record remains highly uncertain due to the complex geological history of our planet.

On Mars, Frey (2008) has identified old impact basins that cluster around crater-density-based ages of about 4.2-4.1 Ga. However, these crater ages may be an overestimate as they are based on the assumption that there was a smooth decline in the impact rate since the origin of the solar system, $\sim 4.5 \mathrm{Ga}$.

The exact onset age and the duration of the LHB are a subject of current debate (e.g., Chapman et al. (2007)). In a recent paper, Norman \& Nemchin (2014) report a large basin-scale melting event on the Moon at $4.22 \pm 0.01 \mathrm{Ga}$, based on new measurements of $\mathrm{U}-\mathrm{Pb}$ isotopic compositions in a lunar melt rock sample; they suggest an earlier onset of the basin-forming epoch that was more prolonged and less intense than inferred from previous lunar sample studies. This interpretation of the impact chronology of the inner solar system attributes the concentration of lunar highland impact melt and breccia ages at about 3.9-3.7 Ga to a sampling bias.

The possibility that the Apollo lunar samples suffer from a sampling bias and reflect the age of a single large basin-forming impact, the Imbrium basin, has been discussed in the lunar literature (e.g., Haskin et al. (1998)). A recent study of zircons from the Apollo 12 landing site finds that the Imbrium impact occurred $3.92 \pm 0.013 \mathrm{Ga}$ (Liu et al., 2012). Stöffler et al. (2006) reviewed the radiometric ages of Apollo samples and compiled a list of the ages of lunar highlands impact breccias and melts, including clast-poor impact melts (10 samples), crystalline melt breccias ( 21 samples), fragmental breccias ( 3 samples) and granulitic breccias and granulites (10 samples). All of these ages lie between 3.7 and $4.2 \mathrm{~Gy}$. We note that, of the 45 samples' ages listed by Stöffler et al. (2006), 29 (64\%) have error bars outside of the Imbrium impact age of 3.92 Gyr. Most of these (90\%) are less than the age of the Imbrium impact (see Figure A-2 in the Appendix). These data indicate that most of the lunar impact breccia and melts are not related to the Imbrium impact.

Some recent studies argue that the LHB may have extended to much more recent times than 3.8 or 3.7 $\mathrm{Ga}$ in the inner Solar system. An analysis of impact generated spherules in pre-Cambrian terrestrial sediments indicates that large basin-forming impacts continued on Earth much longer than previously thought, possibly up to $\sim 2 \mathrm{Ga}$ (Johnson \& Melosh, 2012). Bottke et al. (2012) have suggested, based on a dynami- 
cal model of the orbital migration history of the giant planets, that the LHB began $4.1 \mathrm{Ga}$ and continued to produce basin-size craters upto at least $\sim 2.5 \mathrm{Ga}$; they suggest that most of the late impactors could have originated in an extended and now largely extinct portion of the asteroid belt between 1.7 and $2.1 \mathrm{AU}$, a so-called E-belt. From the impact flux estimates of Bottke et al. (2012)'s model (ten lunar basins between 3.7 Ga and 4.1 Ga, fifteen terrestrial basins between $2.5 \mathrm{Ga}$ and $3.7 \mathrm{Ga}$ ), and adopting their 17:1 ratio of the gravitational cross section of Earth and Moon, it is straightforward to calculate the ratio of the modeled average impactor flux during the LHB epoch (4.1-3.7 Ga) to that during the extended LHB epoch (2.5-3.7 $\mathrm{Ga})$ :

$$
\frac{\text { average impactor flux (LHB) }}{\text { average impactor flux (extended LHB) }}=\frac{17 \times 10 /(4.1-3.7)}{15 /(3.7-2.5)}=34 \text {. }
$$

This implies that the extended LHB impact flux was very significantly smaller than the peak LHB flux. The crater record provides additional useful constraints for this extended-LHB model, as we discuss below.

We know from the post-Orientale crater counts that the LHB was still occurring after the Orientale impact, but at a rate about 6.5 times less than the peak period (see Figure 57. Based on crater counts and radiometric ages of lunar samples, the age of the lunar maria is from 3.9 to $1.2 \mathrm{Ga}$ with the greatest lava eruption volume occurring between about $3.3 \mathrm{Ga}$ and $3.7 \mathrm{Ga}$ (Hiesinger et al., 2000, 2003). The superposed craters on the maria have a size distribution consistent with Population 2 (see Figure 4). Therefore, the impact rate of the LHB extension must have been low enough that the accumulation of Population 2 masked the later stages of Population 1 impacts. Since Population 1 is deficient in smaller craters $(<50 \mathrm{~km}$ diameter) compared to Population 2 (see Figures 1 and 2), this would result in much less modification of Population 2 at smaller diameters. Furthermore, the formation of parts of the lunar maria during the time interval $3.9 \mathrm{Ga}$ to $2.0 \mathrm{Ga}$ would have destroyed some of the later Population 1 craters. As a simple illustration, we show in Figure 16 the results of a simulation of the effects of a $99 \%$ reduction of the impact flux of the LHB between $3.9 \mathrm{Ga}$ and $2.0 \mathrm{Ga}$ and its combination with the post mare Population 2 craters. The resulting crater size distribution is similar to the post-mare Population 2 curve in both shape and magnitude of crater density. The power law fits to the curves are also similar in slope and magnitude, but the "Combination curve is at a slightly higher density and slightly greater slope than the post-mare curve. This indicates that the LHB impact flux was reduced by $\sim 99 \%$ or more between $3.9 \mathrm{Ga}$ and $2.0 \mathrm{Ga}$.

Another recent study, Morbidelli et al. (2012), employs cosmogonic models of the ancient asteroidal population and its dynamical evolution to argue that the LHB began as an uptick of a factor of 5-to-10 in the bombardment rate at $4.1 \mathrm{Ga}$ and decayed with an exponential timescale of $\sim 144$ myr. This differs only slightly from the timeline of the 'terminal lunar cataclysm' of an impact flux spike during 4.0-3.8 Ga inferred by Tera et al. (1974) and others based on radiometric analyses of lunar samples. The crater SFDs presented here do not conclusively distinguish between the two timelines. We can only note that the crater record indicates that the transition from Population 1 to Population 2 projectiles was quite complete by about 3.7 Ga because Population 1 is absent on the lunar maria that formed since that time. 
The uncertainty of the onset age and the duration of the LHB do not affect our main result of two populations of impactors and their association with the LHB and the post-LHB bombardment in the inner solar system.

\subsection{Dynamical Mechanism for the LHB}

The congruence of the size distribution of the projectiles of Population 1 craters and of the MBAs indicates a dynamical ejection process that was largely insensitive to the asteroid mass, and very distinct from the dynamical mechanism that produced Population 2. Strom et al. (2005) suggested that Population 1 could be identified with the LHB, and that the source of the LHB impactors was the main asteroid belt. The LHB impact spike could plausibly have been caused by the size-independent dynamical ejection of main belt asteroids during a short-duration orbital migration of the giant planets.

The orbital migration of the giant planets was previously proposed to explain the orbit of Pluto and to predict the orbital distribution in the Kuiper Belt (Malhotra, 1993, 1995) and to explain the relative paucity of asteroids in the outer asteroid belt (Liou \& Malhotra, 1997). The hypothesis of giant planet migration has subsequently been supported with discoveries in the Kuiper Belt as well as subsequent theoretical studies (cf. Morbidelli et al., 2009). With regard to the main asteroid belt, it has long been noted that there exist many 'gaps', known as the 'Kirkwood Gaps' (Kirkwood, 1882), near the locations of many mean motion resonances with Jupiter and the $\nu_{6}$ secular resonance associated with Saturn's mean perihelion precession rate. The dynamical effect of these gravitational resonances is that they cause orbital instabilities over certain limited ranges of semimajor axis in the main asteroid belt. The orbital migration of Jupiter and Saturn would have caused these unstable zones to sweep across a range of asteroid semimajor axes that were previously populated with asteroids, thereby causing asteroids to be ejected from the main belt into planet-crossing orbits. Indeed, the de-biased orbital distribution of the main asteroid belt reveals that the extent of the Kirkwood gaps and the density of asteroids near the $\nu_{6}$ secular resonance cannot be explained with the perturbations of the giant planets in their current orbits, but can be accounted for only if Jupiter has migrated inward by $\sim 0.2 \mathrm{AU}$ and Saturn has migrated outward by $\sim 1$ AU (Minton \& Malhotra, 2009). Furthermore, from the eccentricity distribution of main belt asteroids, it is inferred that the timescale of Jupiter and Saturn's migration was possibly as short as a few million years (Minton \& Malhotra, 2011). If planet migration is the correct explanation, then one also needs to explain its short timescale as well as the nearly 600 million year delay between the formation of the giant planets and their orbital migration. Such an explanation has been proposed by Tsiganis et al. (2005) with a scenario known as the "Nice model". In this scenario, the giant planets initially form in a marginally stable orbital configuration and migrate very slowly for the first few hundred million years, until such time as Jupiter and Saturn encounter a 2:1 mean motion resonance. This planet-planet resonant encounter causes a strong chaotic episode in the orbital evolution of all the giant planets, changing their orbital eccentricities and triggering a fast migration. This causes a major dynamical instability in both the Kuiper Belt and the asteroid belt, and, therefore, a spike in the impact flux 
of both comets and asteroids on the inner Solar system planets and the Moon (Gomes et al., 2005). The authors estimate that the cataclysmic bombardment lasted 30-150 million years, and that comet impacts dominated at early times and asteroid impacts dominated at later times during the impact spike. Additional work is needed to fully test this model and to constrain its free parameters (e.g. Dawson \& Murray-Clay, 2012; Agnor \& Lin, 2012).

An alternative interpretation is offered by Ćuk et al. 2010, 2011); Ćuk 2012). These authors argue that the transition from Population 1 impactors to Population 2 impactors occurred prior to the formation of the Imbrium and Orientale basins, i.e., prior to $\sim 3.8 \mathrm{Ga}$, and therefore the LHB must be associated with Population 2, rather than Population 1 craters. Ćuk (2012) presents the following scenario. The Population 1 craters were made over an extended period of time prior to $\sim 3.8 \mathrm{Ga}$, and the source of their impactors was a very large primordial population of Mars-crossing asteroids that decayed gradually over several hundred million years. The LHB was caused by the singular break-up at $\sim 3.8 \mathrm{Ga}$ of a Vesta-size body in this population. The size distribution of the break-up fragments is postulated to be similar to that of Population 2. Some basic aspects of this scenario are consistent with the crater record as we understand it: the existence of two different impactor populations, and the similarity of the ancient Population 1 with the size distribution of the main belt asteroids (very plausibly the putative primordial Mars-crossing asteroid population as well as the E-belt shared the main belt size distribution). But some aspects directly contradict the data as we understand it. Population 2 craters do not dominate the Imbrian and post-Orientale craters (see Figure 5 . also Malhotra \& Strom (2011)). Second, there is a conflict between Population 2 being that of a largeasteroid-break-up event causing a short-lived LHB and the evidence that the Population 2 SFD has been in near-steady-state over the past $\sim 3.8$ gigayears of the post-LHB crater record. Third, the expected fragment size distribution of asteroid break-up events, based on observations of asteroid collisional families and on numerical simulations of family-formation, is quite different than the SFD of either Population 1 or Population 2 projectiles (e.g. Benavidez et al. 2012). A large-asteroid-break-up event as a dynamical cause of the LHB has also been investigated previously by Zappalà et al. (1998) and Ito \& Malhotra (2006); the latter work concluded that this was not a viable mechanism because it requires an implausibly large asteroid parent body. For these reasons, this scenario is not supported by the data as we understand it.

\subsection{Implications for Age Dating from the Impact Crater Record}

If the Late Heavy Bombardment is the result of a cataclysmic event, as the evidence indicates, then the previous cratering record has been significantly obliterated, and the ancient impact flux (prior to about $4 \mathrm{Ga}$ ) is presently unknown. Therefore, our current knowledge of the impact flux history in the inner Solar system from the impact crater record is not adequate to date surfaces older than about 3.9 billion years. Studies that claim to date surfaces older than this date from the cratering record of Population 1 are only dating them between about 3.8 and 4.0 billion years. 
Surfaces that display Population 2 craters are younger than about 3.7 billion years and can be dated relatively reliably by using the NEO flux at the planet in question (e.g. Le Feuvre \& Wieczorek, 2011). There may be impact craters formed during the extended LHB as discussed earlier in which case the derived model age will be an upper limit. Although comet impacts are surely contained within the Population 2 crater population, they have not been abundant enough to affect the SFD. Therefore, Population 2 must be dominated by asteroid impacts, unless comet impacts produce the same crater SFD as NEOs. However, ages derived from the NEO flux are upper limits because some comet impacts are probably present.

The small-crater population (approximately $D<1 \mathrm{~km}$ diameter on the Moon and Mars, $D>10 \mathrm{~km}$ on Mercury) should be used with great caution to date surfaces because it is contaminated by large numbers of secondary impact craters (McEwen \& Bierhaus, 2006; Robbins \& Hynek, 2011; Xiao \& Strom, 2012). This is particularly true for Mercury where the secondaries are larger for a given size crater than anywhere else in the Solar system (Xiao et al., 2014). Some craters on Mercury have more circular secondaries, rendering the distinguishing of primaries and secondaries more difficult than on the other planets (Xiao et al., 2014). Mercury basin secondaries begin to affect the crater SFD at diameters of about 9-10 km in almost all heavily cratered areas of the planet (Strom et al., 2011).

\section{SUMMARY}

Two populations of objects of distinctly different size-frequency distributions have impacted the inner Solar system planets and the Moon. When combined with the accumulated data on the age-dating of lunar and meteorite samples, as well as insights from Solar system dynamics, the simplest interpretation is the following. One population is responsible for the Late Heavy Bombardment and the other is responsible for impacts after the Late Heavy Bombardment. The population responsible for the Late Heavy Bombardment originated from Main Belt Asteroids while the younger population originated from Near Earth Objects. That the size distribution of the projectiles responsible for the Late Heavy Bombardment is the same as Main Belt Asteroids means that they were ejected in a size-independent manner by means of a gravitational instability. A plausible cause was the orbital migration of Jupiter and Saturn causing a sweeping of gravitational resonances through the Main Asteroid Belt and resulting in a cataclysmic bombardment of the inner Solar system. The younger population is also derived from the Main Asteroid Belt, but ejected by the size-dependant Yarkovsky effect that gradually feeds asteroids into unstable gravitational resonances; we observe the source of these impactors at the present time as the NEOs. Figure 17 is a diagram to illustrate in a very general way the impact history of the inner Solar system. Surfaces younger than about 3.7 Ga can be dated in a relatively reliable way by measuring crater densities and using estimates of the near-planet aster-

oid impact flux at the appropriate planet. But this technique must be applied in crater diameter ranges larger than those of Population S (secondaries). These ages will be upper limits because some comet impacts and extended LHB impacts are possibly present in Population 2 craters. The ancient crater record prior to the 
LHB has been significantly obliterated, and ancient surfaces cannot be reliably age-dated from the cratering record.

Acknowledgements We thank Z. Ivezic and J. S. Stuart for providing us with digital versions of their published data. RM acknowledges research support from NSF grant \#AST-1312498. We also thank the anonymous referee for comments which improved the quality of this paper. 


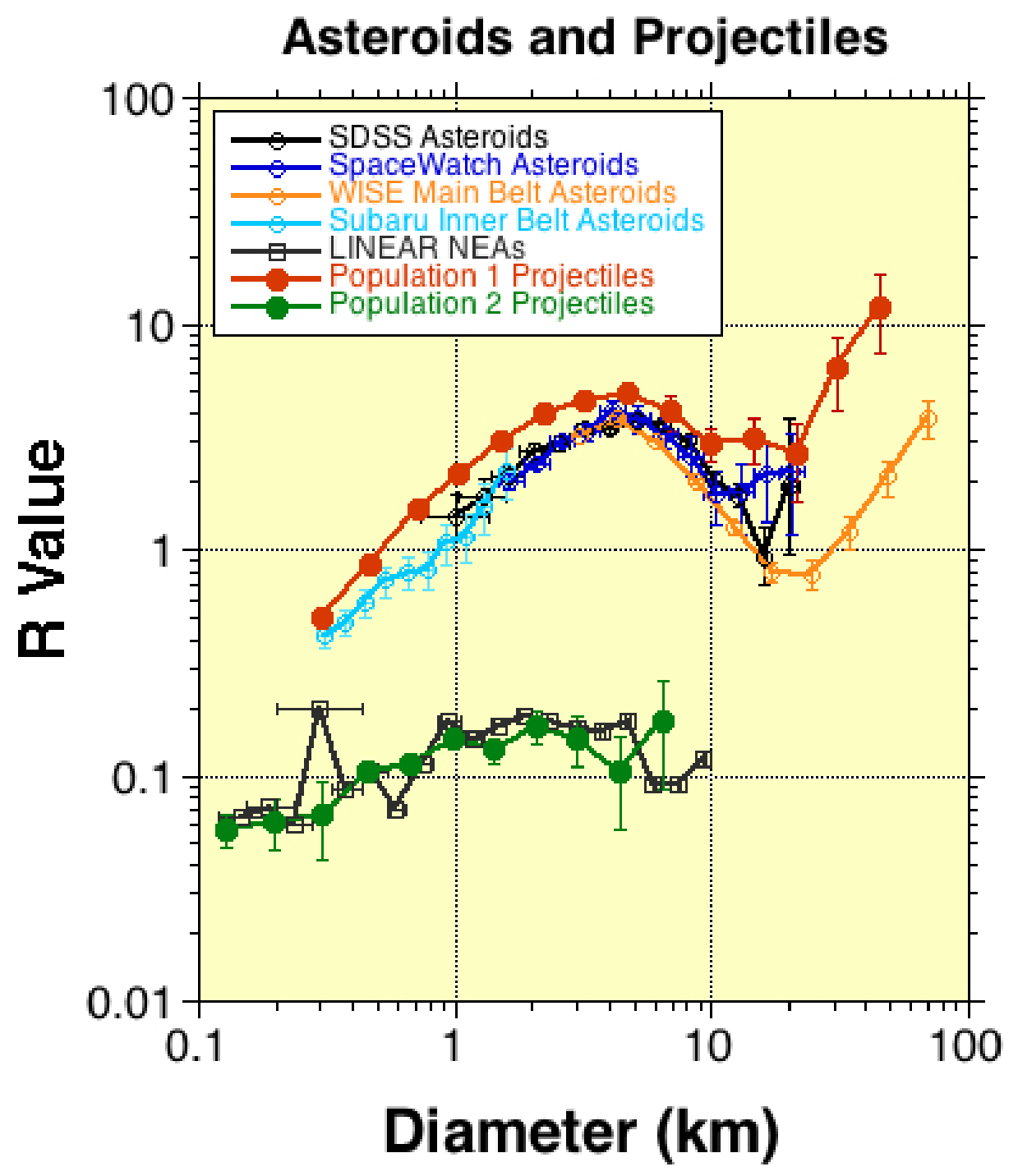

Fig. 14 The SFDs of the impactors derived from the crater SFDs compared with those of Main Belt Asteroids and Near Earth Objects. The red points are impactors derived from the lunar highland crater distribution (Population 1; Figure 1), and the green points are derived from the young Mars plains crater population (Population 2; Figure 1). The vertical positions of the asteroid R-plots are arbitrary; the scale factor is chosen for clarity of comparison. See text for detailed explanation. 


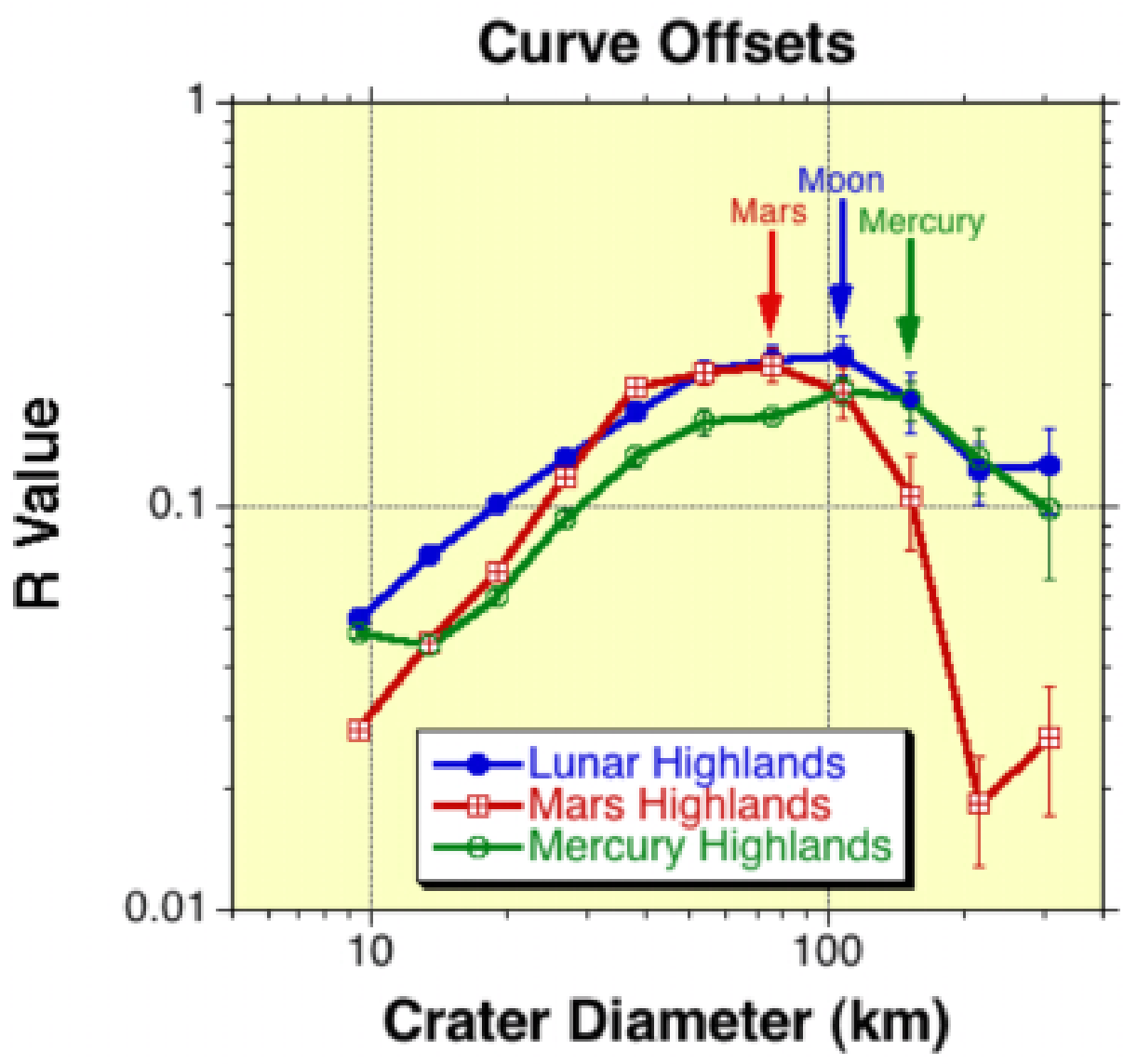

Fig. 15 Displacement of the crater SFD curves attributed to differences in the median asteroid impact velocities at Mercury, the Moon and Mars. The arrows indicate the "downturn" location of each curve. The locations of the peaks are also similarly displaced. See text for detailed explanation. 


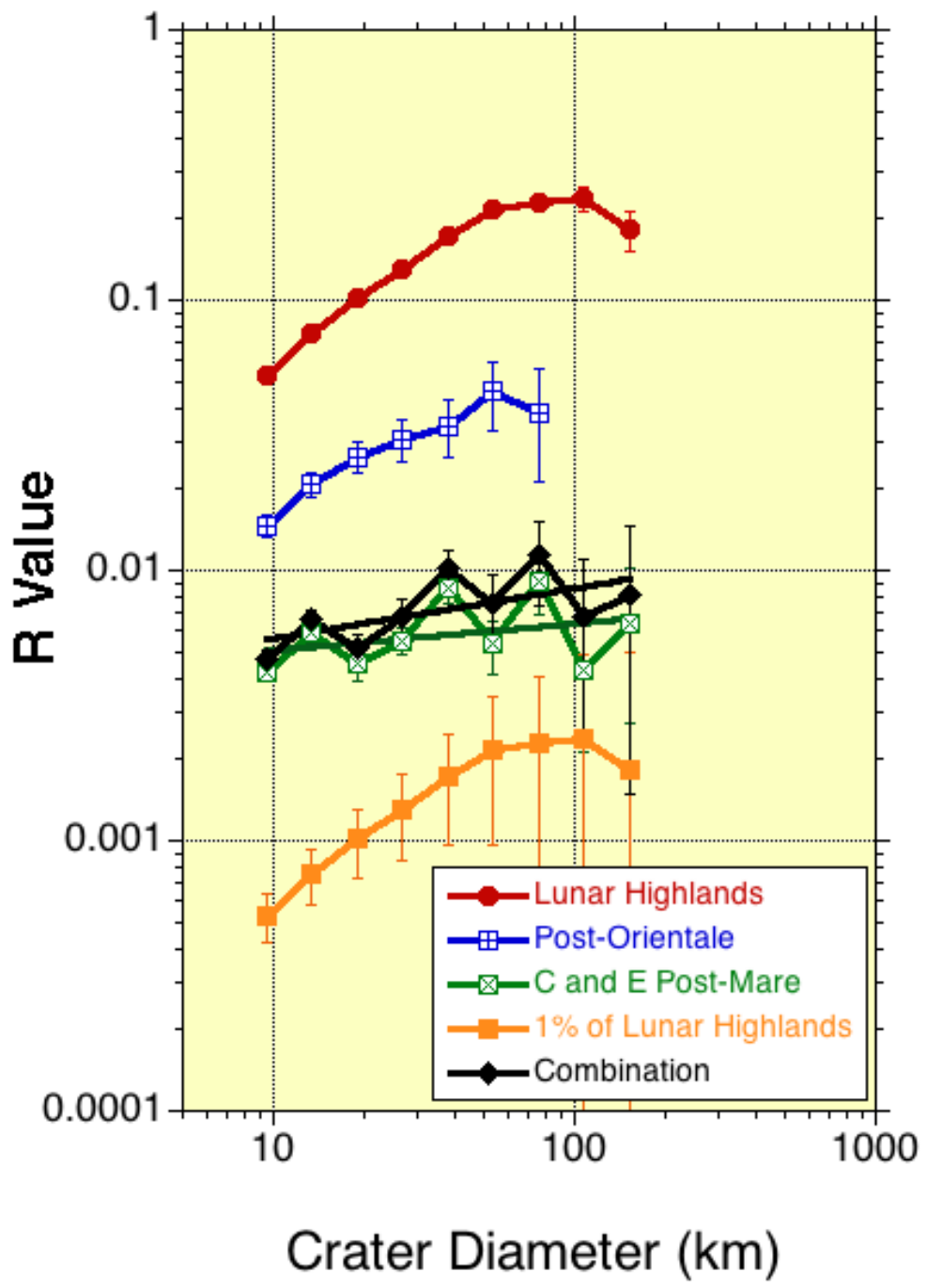

Fig. 16 The top curve (red) shows the lunar highlands $R$ plot and the blue curve shows the postOrientale $R$ plot. The green curve is the $R$ plot for the post-mare Copernican and Eratosthenian craters; the green straight line is a best-fit power law. The orange curve shows a simulated lunar highlands crater density curve reduced by $99 \%$. The "Combination" curve (black) is the combination of post-mare Copernican and Etatosthenian craters and 1\% the lunar highlands; the black straight line is a best-fit power law. 


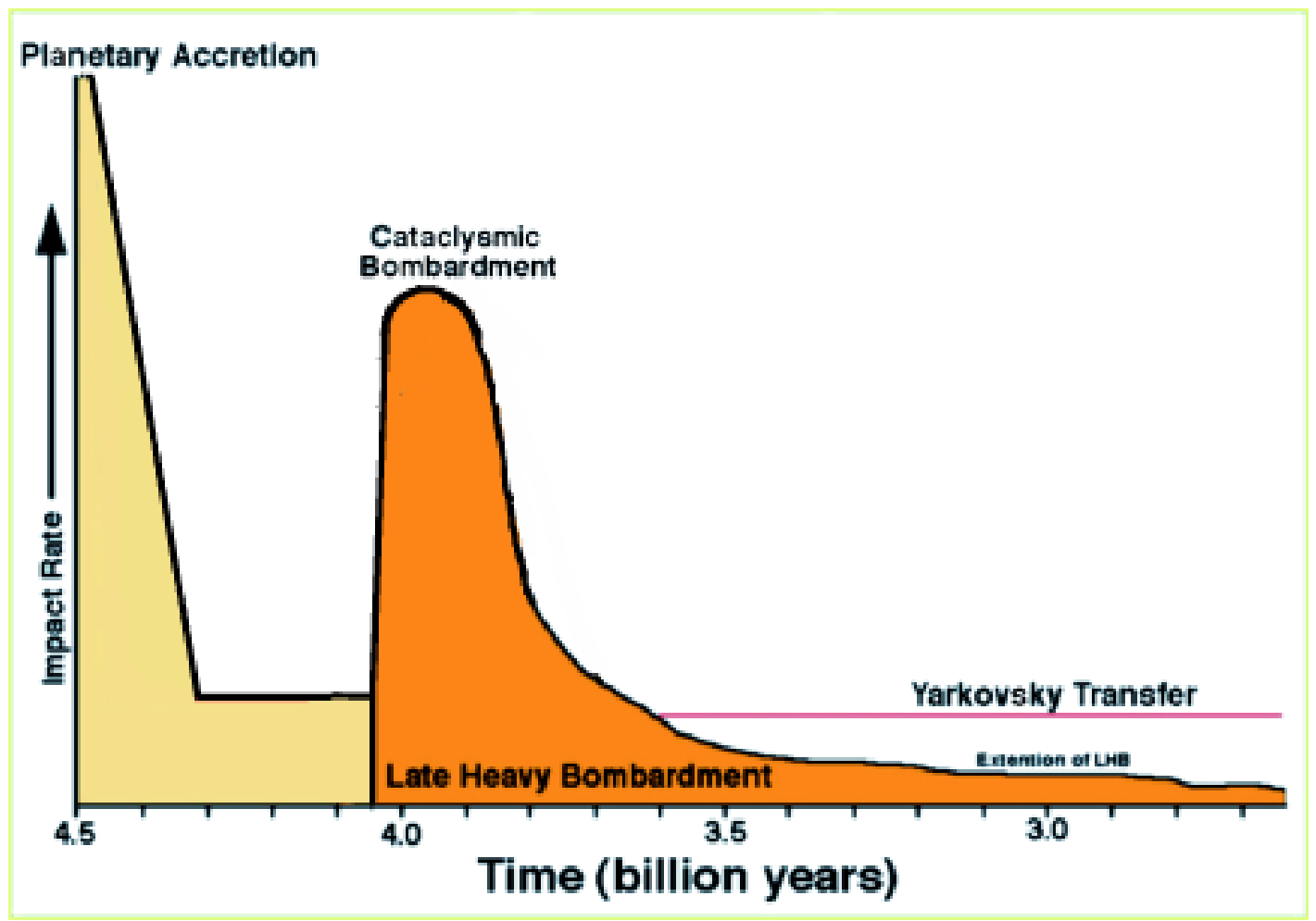

Fig. 17 Schematic diagram of the impact history of the inner Solar system. The high impact rate near 4.5 Ga represents planetary accretion that formed the terrestrial planets. The steep drop in the impact rate just after $4.5 \mathrm{Ga}$ represents the fast collisional and dynamical loss of the local impactor population after planet formation. The LHB began at some time prior to about $3.9 \mathrm{Ga}$, as indicated by the evidence presented in sections 3 and 4 The red horizontal lines between about 4.4 and 4.1 billion years and after $\sim 3.6$ billion years represent impacts by means of Yarkovsky transfer of asteroids from the asteroid belt; the former is higher than after the LHB because there were many more asteroids at that time. The total number of impacts via Yarkovsky transfer after the main period of the LHB would have masked the putative extended LHB (see section 4.2 and Figure 16. 


\section{References}

Agnor, C. B., \& Lin, D. N. C. 2012, ApJ, 745, 143

Arthur, D.W.G., Agnieray, A.P., Horvath, R.A., Wood, C.A., \& Chapman, C.R. 1964, Comm. Lunar Planet. Lab., 2, 71

Benavidez, P.G., Durda, D.D., Enke, B.L., et al. 2012, Icarus, 219, 57

Bottke, W. F., Durda, D. D., Nesvorný, D., et al. 2005, Icarus, 175, 111

Bottke, W. F., Levison, H. F., Nesvorný, D., \& Dones, L. 2007, Icarus, 190, 203

Bottke, W. F., Vokrouhlický, D., Minton, D., et al. 2012, Nature, 485, 78

Bottke, W. F., Vokrouhlický, D., Rubincam, D. P., \& Nesvorný, D. 2006, Annu. Rev. Earth. Planet. Sci., 34, 157

Chapman, C. R., Cohen, B. A., \& Grinspoon, D. H. 2007, Icarus, 189, 233

Chapman, C. R., \& McKinnon, W. B. 1986, Satellites, 492

Cheng, A. F. 2004, Icarus, 169, 357

Collins, G. S., Melosh, H. J., \& Marcus, R. A. 2005, Meteoritics \& Planetary Science, 40, 817

Crater analysis techniques working group 1979, Icarus, 37, 467

Croft, S.K. 1985, J. Geophys. Res., 90, C828, originally appeared in Proc. 15th Lunar Planet. Sci. Conf.

Ćuk, M. 2012, Icarus, 218, 69

Ćuk, M., Gladman, B. J., \& Stewart, S. T. 2010, Icarus, 207, 590

Ćuk, M., Gladman, B. J., \& Stewart, S. T. 2011, Icarus, 216, 363

Dawson, R. I., \& Murray-Clay, R. 2012, ApJ, 750, 43

Dones, L., Chapman, C.R., McKinnon, W.B., et al. 2009, in Saturn from Cassini-Huygens, 613-635 (Berlin: Springer)

Dundas, C.M., \& McEwen, A.S. 2007, Icarus, 186, 31

Farinella, P., \& Vokrouhlicky, D. 1999, Science, 283, 1507

Fassett, C. I., Kadish, S. J., Head, J. W., Solomon, S. C., \& Strom, R. G. 2011, Geophys. Res. Lett., 38, L10202

Feldman, W.C., Gasnault, O., Maurice, S., et al. 2002, J. Geophys. Res., 107, 10.1029/2001JE001506

French, B.M. 1998, Traces of catastrophe: A handbook of shock-metamorphic effects in terrestrial meteorite impact structures (Houston: Lunar and Planetary Institute)

Frey, H. 2008, Geophys. Res. Lett., 35, 13203

Gallant, J., Gladman, B., \& Ćuk, M. 2009, Icarus, 202, 371

Gladman, B. J., Migliorini, F., Morbidelli, A., et al. 1997, Science, 277, 197

Gomes, R., Levison, H. F., Tsiganis, K., \& Morbidelli, A. 2005, Nature, 435, 466

Grieve, R. A. F., \& Shoemaker, E. M. 1994, in Hazards Due to Comets and Asteroids, edited by T. Gehrels, M. S. Matthews, \& A. M. Schumann, 417

Hartmann, W. 1995, Meteoritics, 30, 451 
Hartmann, W. K. 1966, Icarus, 5, 406

Hartmann, W. K. 2005, Icarus, 174, 294

Hartmann, W. K., Quantin, C., \& Mangold, N. 2007, Icarus, 186, 11

Hartmann, W.K., \& Neukum, G. 2001, Space Sci. Rev., 96, 165

Haskin, L. A., Korotev, R. L., Rockow, K. M., \& Jolliff, B. L. 1998, Meteoritics and Planetary Science, 33, 959

Head, J. W., Fassett, C. I., Kadish, S. J., et al. 2010, Science, 329, 1504

Head, J.W., Chapman, C.R., Strom, R.G., et al. 2011, Science, 333, 1853

Hiesinger, H., Head, J.W.III., Wolf, U., Jaumann, R., \& Neukum, G. 2003, J. Geophys. Res., 108, 5065

Hiesinger, H., Jaumann, R., Neukum, G., \& Head, J.W.III. 2000, J. Geophys. Res., 105, 29239

Ito, T., \& Malhotra, R. 2006, Adv. Space Res., 38, 817

Ito, T., \& Malhotra, R. 2010, Astron. Astrophys., 519, A63

Ivanov, B. A., Neukum, G., Bottke, W. F., \& Hartmann, W. K. 2002, Asteroids III, 89

Ivezić, Ž., Tabachnik, S., Rafikov, R., et al. 2001, AJ, 122, 2749

Jedicke, R., \& Metcalfe, T. S. 1998, Icarus, 131, 245

JeongAhn, Y., \& Malhotra, R. 2010, in AAS/Division for Planetary Sciences Meeting Abstracts \#42,

Bulletin of the American Astronomical Society, vol. 42, 1053-+

Johnson, B. C., \& Melosh, H. J. 2012, Nature, 485, 75

Joy, K.H., Zolensky, M.E., Nagashima, K., et al. 2012, Science, 336, 1426

Kirchoff, M. R., Chapman, C. R., Marchi, S., et al. 2013, Icarus, 225, 325

Kirkwood, D. 1882, The Observatory, 5, 194

Kring, D. A., \& Cohen, B. A. 2002, JGR, 107, 5009

Le Feuvre, M., \& Wieczorek, M. A. 2011, Icarus, 214, 1

Liou, J. C., \& Malhotra, R. 1997, Science, 275, 375

Liu, D., Jolliff, B. L., Zeigler, R. A., et al. 2012, Earth and Planetary Science Letters, 319, 277

Malhotra, R. 1993, Nature, 365, 819

Malhotra, R. 1995, AJ, 110, 420

Malhotra, R., \& Strom, R. G. 2011, Icarus, 216, 359

Marchi, S., Chapman, C. R., Fassett, C. I., et al. 2013, Nature, 499, 59

Marchi, S., Mottola, S., Cremonese, G., Massironi, M., \& Martellato, E. 2009, AJ, 137, 4936

Masiero, J. R., Mainzer, A. K., Grav, T., et al. 2011, ApJ, 741, 68

McEwen, A. S., \& Bierhaus, E. B. 2006, Annual Review of Earth and Planetary Sciences, 34, 535

McEwen, A.S., Preblich, B.S., Turtle, E.P., et al. 2005, Icarus, 176, 351

McKinnon, W.B., Chapman, C.R., \& Housen, K.R. 1991, in Uranus, edited by J.T. Bergstralh, E.D. Miner, \& M.S. Matthew, 629-692 (Tucson, Arizona: The University of Arizona Press) 
Melosh, H. J. 1989, Impact cratering: A geologic process (New York, New York: Oxford University Press), 1st edn.

Melosh, H.J., \& Beyer, R.A. 1999, Computing Projectile Size from Crater Diameter, http://www.lpl.arizona.edu/tekton/crater_p.html

Michael, G. G. 2013, Icarus, 226, 885

Michael, G.G., \& Neukum, G. 2010, Earth Planet. Sci. Lett., 294, 223

Minton, D. A., \& Malhotra, R. 2009, Nature, 457, 1109

Minton, D. A., \& Malhotra, R. 2010, Icarus, 207, 744

Minton, D. A., \& Malhotra, R. 2011, ApJ, 732, 53

Morbidelli, A., Bottke, W. F., Jr., Froeschlé, C., \& Michel, P. 2002, Asteroids III, 409-422

Morbidelli, A., Brasser, R., Tsiganis, K., Gomes, R., \& Levison, H. F. 2009, A\&A, 507, 1041

Morbidelli, A., Marchi, S., Bottke, W. F., \& Kring, D. A. 2012, Earth and Planetary Science Letters, 355, 144

Morbidelli, A., \& Vokrouhlický, D. 2003, Icarus, 163, 120

Morota, T., \& Furumoto, M. 2003, Earth and Planetary Science Letters, 206, 315

Neukum, G., \& Ivanov, B. A. 1994, in Hazards Due to Comets and Asteroids, 359-416

Neukum, G., Ivanov, B. A., \& Hartmann, W. K. 2001, Space Science Reviews, 96, 55

Norman, M. D., \& Nemchin, A. A. 2014, Earth and Planetary Science Letters, 388, 387

Norman, M.D., Borg, L.E., Nyquist, L.E., \& Bogard, D.D. 2003, Meteorit. Planet. Sci., 38, 645

Robbins, S.J., \& Hynek, B.M. 2011, Geophys. Res. Lett., 38, L05201

Rubincam, D.P. 1988, J. Geophys. Res., 93, 13805

Ryder, G. 2002, JGR, 107, 5022

Schmidt, R. M., \& Housen, K. R. 1987, Int. J. Impact Engng., 5, 543

Scott, D. H., \& Carr, M. H. 1978, United States Geological Survey Misc. Invest. Ser. (Map I-1083, scale $1: 25,000,000),-$,

Shoemaker, E.M. 1965, in Technical Report, vol. 32-700, 75-134 (JPL/NASA), ranger 7, Part 2, Experimenters' analyses and interpretations

Stern, S.A., \& Durda, D.D. 2000, Icarus, 143, 360

Stöffler, D., Ryder, G., Ivanov, B.A., et al. 2006, Reviews Minerology \& Geochem, 60, 519

Strom, R. G., Banks, M. E., Chapman, C. R., et al. 2011, Planet. Space Sci., 59, 1960

Strom, R. G., Chapman, C. R., Merline, W. J., Solomon, S. C., \& Head, J. W. 2008, Science, 321, 79

Strom, R. G., Malhotra, R., Ito, T., Yoshida, F., \& Kring, D. A. 2005, Science, 309, 1847

Strom, R. G., Schaber, G. G., \& Dawsow, D. D. 1994, JGR, 99, 10899

Strom, R.G. 1979, Space Sci. Rev., 24, 3

Strom, R.G., Croft, S.K., \& Boyce, J.M. 1990, Science, 250, 437 
Strom, R.G., \& Neukum, G. 1988, in Mercury, edited by F. Vilas, C.R. Chapman, \& M.S. Mathhews, 363373 (Tucson, Arizona: The University of Arizona Press)

Strom, R.G., Woronow, A., \& Gurnis, M. 1981, J. Geophys. Res., 862, 8659

Stuart, J. S., \& Binzel, R. P. 2004, Icarus, 170, 295

Tera, F., Papanastassiou, D. A., \& Wasserburg, G. J. 1973, Abstracts of the Lunar and Planetary Science Conference, 4,723

Tera, F., Papanastassiou, D. A., \& Wasserburg, G. J. 1974, Earth and Planetary Science Letters, 22, 1

Trail, D., Mojzsis, S. J., \& Harrison, T. M. 2007, Geochimica et Cosmochimica Acta, 71, 4044

Tsiganis, K., Gomes, R., Morbidelli, A., \& Levison, H. F. 2005, Nature, 435, 459

Turner, G., Cadogan, P. H., \& Yonge, C. J. 1973, LPSCIV, 4, 1889

Turner, G., Knott, S.F., Ash, R.D., \& Gilmour, J.D. 1997, Geochim. Cosmochim. Acta, 61, 3835

Usui, F., Kasuga, T., Hasegawa, S., et al. 2013, Astrophys. J., 762, 56

Wilhelms, D. E., Oberbeck, V. R., \& Aggarwal, H. R. 1978, 9, 3735

Willbold, M., Elliott, T., \& Moorbath, S. 2011, Nature, 477, 193

Wood, C.A., \& Anderson, L. 1978, Proc. Lunar Planet. Sci. Conf., 9, 3669

Xiao, Z., Strom, R. G., Chapman, C. R., et al. 2014, Icarus, 228, 260

Xiao, Z., \& Strom, R.G. 2012, Icarus, 220, 254

Yoshida, F., Nakamura, T., Watanabe, J.-I., et al. 2003, Publications of the Astronomical Society of Japan, 55,701

Zahnle, K.J. 1992, J. Geophys. Res., 97, 10243

Zappalà, V., Cellino, A., Gladman, B., Manley, S., \& Migliorini, F. 1998, Icarus, 134, 176 
Appendix

Table A-1: References and Notes for Crater Data

\begin{tabular}{|c|c|c|c|}
\hline $\begin{array}{c}\text { Craters } \\
\text { Description }\end{array}$ & Reference(s) & Figures(s) & Notes \\
\hline $\begin{array}{l}\text { Lunar } \\
\text { Highlands } \\
\text { craters }\end{array}$ & Arthur et al., 1963 & $\begin{array}{l}1,3,4,5,7,9,10,12 \\
13,15,16\end{array}$ & $\begin{array}{l}\text { Lunar front-side } \\
\text { highlands }\end{array}$ \\
\hline $\begin{array}{l}\text { Lunar Class } 1 \\
\text { craters }\end{array}$ & $\begin{array}{l}\text { Strom, 1977; Arthur et } \\
\text { al., } 1963\end{array}$ & 4,6 & $\begin{array}{l}\text { These are both } \\
\text { highland and Mare } \\
\text { craters. }\end{array}$ \\
\hline $\begin{array}{l}\text { Lunar } \\
\text { Copernican and } \\
\text { Eratosthenian }\end{array}$ & Wilhelms et al., 1978. & $4,12,16$ & $\begin{array}{l}\text { These are post-mare } \\
\text { craters identified } \\
\text { both } \\
\text { morphologically and } \\
\text { stratigraphically }\end{array}$ \\
\hline $\begin{array}{l}\text { Lunar Post- } \\
\text { Mare }\end{array}$ & $\begin{array}{l}\text { Strom, 1977; Arthur et } \\
\text { al., } 1963\end{array}$ & 4,16 & $\begin{array}{l}\text { Lunar Front-side } \\
\text { Maria }\end{array}$ \\
\hline $\begin{array}{l}\text { Lunar Post- } \\
\text { Orientale }\end{array}$ & This work & 16 & $\begin{array}{l}\text { Counts on Lunar } \\
\text { Reconnaissance } \\
\text { Orbiter Camera } \\
\text { WAC images }\end{array}$ \\
\hline Lunar Bruno & Xiao \& Strom, 2012 & 11 & \\
\hline $\begin{array}{l}\text { Mercury } \\
\text { Heavily } \\
\text { Cratered }\end{array}$ & $\begin{array}{l}\text { Strom et al., 2008; Strom, } \\
1977\end{array}$ & 1 & $\begin{array}{l}\text { These counts are } \\
\text { from the heavily } \\
\text { crater terrain from } \\
\text { Mariner } 10 \\
\text { incoming, outgoing } \\
\text { and south polar } \\
\text { areas. }\end{array}$ \\
\hline $\begin{array}{l}\text { Mercury } \\
\text { Heavily } \\
\text { Cratered High } \\
\text { Density }\end{array}$ & Strom et al., 2011 & 7 & $\begin{array}{l}\text { This area is a region } \\
\text { from area with one } \\
\text { of the highest crater } \\
\text { densities on } \\
\text { Mercury }\end{array}$ \\
\hline $\begin{array}{l}\text { Mercury } \\
\text { Northern Plains }\end{array}$ & Ostrach, et al., 2011 & 6 & $\begin{array}{l}\text { These counts are the } \\
\text { entire Northern } \\
\text { Plains from } \\
\text { MESSENGER images }\end{array}$ \\
\hline $\begin{array}{l}\text { Mercury Caloris } \\
\text { Exterior Plains }\end{array}$ & Strom et al., 2008, 2011 & 6,10 & $\begin{array}{l}\text { These counts are } \\
\text { from MESSENGER's } \\
\text { first flyby and } \\
\text { Mariner } 10 \text { 's second } \\
\text { encounter. }\end{array}$ \\
\hline Mercury Class 1 & This work & 6,12 & $\begin{array}{l}\text { Same morphological } \\
\text { Class as lunar Class } \\
1 \text { craters }\end{array}$ \\
\hline
\end{tabular}




\section{Appendix \\ Table A-1: References and Notes for Crater Data}

\begin{tabular}{|c|c|c|c|}
\hline $\begin{array}{l}\text { Venus } \\
\text { Production }\end{array}$ & Strom et al, 1994 & 8,12 & $\begin{array}{l}\text { These data come } \\
\text { from the catalog of } \\
\text { Venus craters by } \\
\text { Schaber and Strom }\end{array}$ \\
\hline Venus Screened & Strom et al, 1994 & 8 & As above \\
\hline $\begin{array}{l}\text { Venus Multi- } \\
\text { Screened }\end{array}$ & Strom et al, 1994 & 8 & As above \\
\hline Mars Highlands & Strom et al., 1992 & 9,15 & $\begin{array}{l}\text { These counts are } \\
\text { from the most } \\
\text { heavily craters } \\
\text { regions on Mars as } \\
\text { compiled by Barlow }\end{array}$ \\
\hline $\begin{array}{l}\text { Mars Old } \\
\text { Cratered Plains }\end{array}$ & Strom et al., 1992 & 9,12 & $\begin{array}{l}\text { Moderately cratered } \\
\text { plains east of } \\
\text { Tharsis partly } \\
\text { including the } \\
\text { geologic unit "Old } \\
\text { Volcanic Material" }\end{array}$ \\
\hline $\begin{array}{l}\text { Mars Northern } \\
\text { Plains }\end{array}$ & Strom et al., 1992 & 9 & $\begin{array}{l}\text { This area is largely } \\
\text { Vastitas Borealis } \\
\text { including the } \\
\text { geologic unit } \\
\text { "Mottled Plains } \\
\text { Unit" }\end{array}$ \\
\hline $\begin{array}{l}\text { Mars Hellas } \\
\text { Plains }\end{array}$ & Strom et al., 1992 & 9 & $\begin{array}{l}\text { Plains within the } \\
\text { Hellas Basin }\end{array}$ \\
\hline $\begin{array}{l}\text { Mars Tharsis } \\
\text { Plains }\end{array}$ & Strom et al., 1992 & 9 & $\begin{array}{l}\text { Plains surrounding } \\
\text { the Tharis volcanic } \\
\text { constructs }\end{array}$ \\
\hline $\begin{array}{l}\text { Mars Young } \\
\text { Plains }\end{array}$ & Strom et al., 2005 & $10,11,12$ & $\begin{array}{l}\text { Plains associated } \\
\text { with Tharsis }\end{array}$ \\
\hline $\begin{array}{l}\text { Mars small } \\
\text { rayed craters }\end{array}$ & This work & 11 & $\begin{array}{l}\text { Rayed craters from } \\
\text { Viking orbital } \\
\text { images }\end{array}$ \\
\hline $\begin{array}{l}\text { Callisto and } \\
\text { Ganymede }\end{array}$ & Strom et al. 1981 & 13 & \\
\hline $\begin{array}{l}\text { Rhea and } \\
\text { Tethys }\end{array}$ & This work & 13 & $\begin{array}{l}\text { Previously } \\
\text { Unpublished }\end{array}$ \\
\hline $\begin{array}{l}\text { Ariel, Miranda } \\
\text { and Titania }\end{array}$ & Greenberg et al, 1991 & 13 & \\
\hline Triton & Strom et al., 1990 & 13 & \\
\hline
\end{tabular}




\section{Appendix \\ Figure A-1}

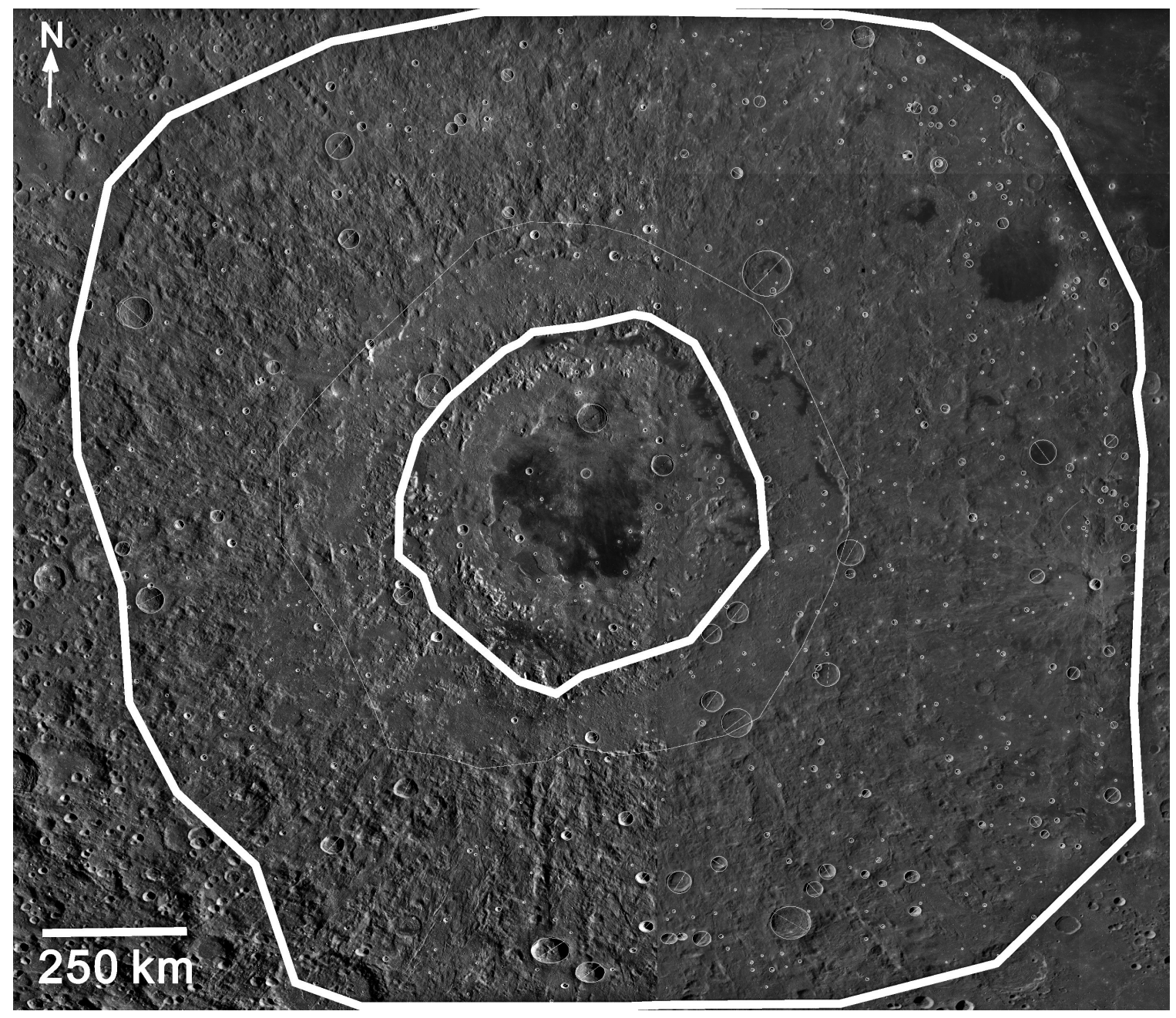

Figure A-1. Crater counts for the Orientale basin. The outer circle denotes the continuous ejecta deposits of the Orientale basin, and the inner circle denote the inner ring of the Orientale basin that has been largely flooded by mare basalts. A slightly thinner circle in the middle of the figure denotes the rim of the Orientale basin. The craters counted are marked in white circles. The base image is from the global monochrome mosaic of the Moon taken from the Lunar Reconnaissance Orbiter Camera (Robinson et al., 2010). The image is in equirectangular projection and the resolution is $100 \mathrm{mi} / \mathrm{pixel}$. The reader should copy and enlarge the image to better see the measured craters. 


\section{Appendix \\ Figure A-2}

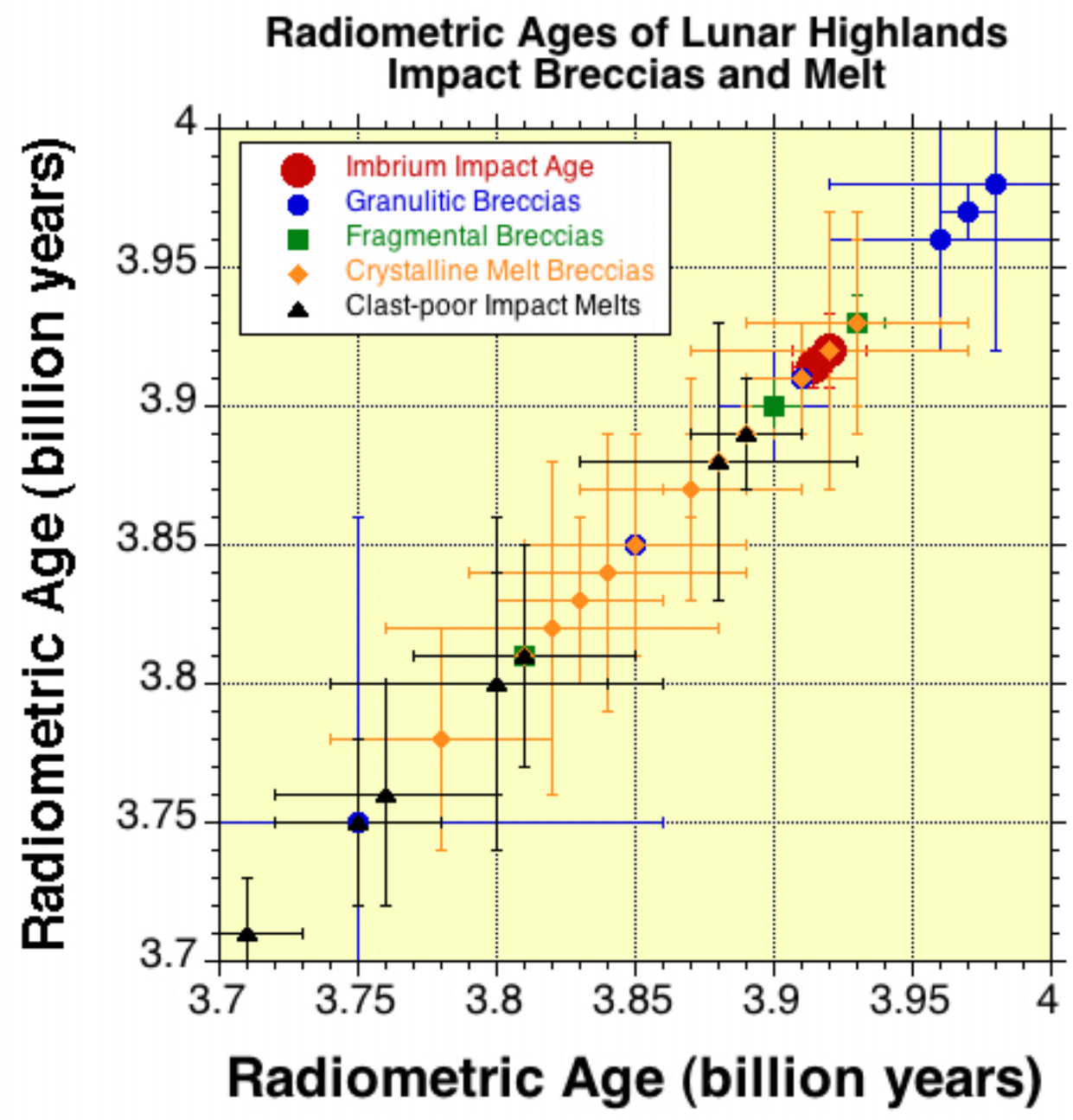

Figure A-2. The radiometric ages of all the 45 lunar highlands impact breccias and melts listed by Stoffer et al. 2006, and the recently determined age of the Imbrium impact by Liu et al (2012). 